\title{
On the Distribution of Penalized Maximum Likelihood Estimators: The LASSO, SCAD, and Thresholding
}

\author{
Benedikt M. Pötscher \\ Department of Statistics, University of Vienna \\ and \\ Hannes Leeb \\ Department of Statistics, Yale University
}

First version: October 2007

This version: March 2009

\begin{abstract}
We study the distributions of the LASSO, SCAD, and thresholding estimators, in finite samples and in the large-sample limit. The asymptotic distributions are derived for both the case where the estimators are tuned to perform consistent model selection and for the case where the estimators are tuned to perform conservative model selection. Our findings complement those of Knight and Fu (2000) and Fan and Li (2001). We show that the distributions are typically highly nonnormal regardless of how the estimator is tuned, and that this property persists in large samples. The uniform convergence rate of these estimators is also obtained, and is shown to be slower than $n^{-1 / 2}$ in case the estimator is tuned to perform consistent model selection. An impossibility result regarding estimation of the estimators' distribution function is also provided.

MSC 2000 subject classification. Primary 62J07, 62J05, 62F11, 62F12, $62 \mathrm{E} 15$.

Key words and phrases. Penalized maximum likelihood, LASSO, SCAD, thresholding, post-model-selection estimator, finite-sample distribution, asymptotic distribution, oracle property, estimation of distribution, uniform consistency.
\end{abstract}

\section{Introduction}

Penalized maximum likelihood estimators have been studied intensively in the last few years. A prominent example is the least absolute selection and shrinkage (LASSO) estimator of Tibshirani (1996). Related variants of the LASSO 
include the Bridge estimators studied by Frank and Friedman (1993), least angle regression (LARS) of Efron, Hastie, Johnston, Tibshirani (2004), or the smoothly clipped absolute deviation (SCAD) estimator of Fan and Li (2001). Other estimators that fit into this framework are hard- and soft-thresholding estimators. While many properties of penalized maximum likelihood estimators are now well understood, the understanding of their distributional properties, such as finite-sample and large-sample limit distributions, is still incomplete. The probably most important contribution in this respect is Knight and $\mathrm{Fu}$ (2000) who study the asymptotic distribution of the LASSO estimator (and of Bridge estimators more generally) when the tuning parameter governing the influence of the penalty term is chosen so that the LASSO acts as a conservative model selection procedure (that is, a procedure that does not select underparameterized models asymptotically, but selects overparameterized models with positive probability asymptotically); see also Knight (2008). In Knight and Fu (2000), the asymptotic distribution is obtained in a fixed-parameter as well as in a standard local alternatives setup. This is complemented by a result in Zou (2006) who considers the fixed-parameter asymptotic distribution of the LASSO when tuned to act as a consistent model selection procedure. Another contribution is Fan and Li (2001) who derive the asymptotic distribution of the SCAD estimator when the tuning parameter is chosen so that the SCAD estimator performs consistent model selection; in particular, they establish the so-called 'oracle' property for this estimator. The results in that latter paper are also fixed-parameter asymptotic results. It is well-known that fixed-parameter (i.e., pointwise) asymptotic results can give a wrong picture of the estimators' actual behavior, especially when the estimator performs model selection; see, e.g., Kabaila (1995), or Leeb and Pötscher (2005, 2008a). Therefore, it is interesting to take a closer look at the actual distributional properties of such estimators.

In the present paper we study the finite-sample as well as the asymptotic distributions of the hard-thresholding, the LASSO (which coincides with softthresholding in our context), and the SCAD estimator. We choose a model that is simple enough to facilitate an explicit finite-sample analysis that showcases the strengths and weaknesses of these estimators in a readily accessible framework. Yet, the model considered here is rich enough to demonstrate a variety of phenomena that will also occur in more complex models. We study both the cases where the estimators are tuned to perform conservative model selection as well as where the tuning is such that the estimators perform consistent model selection. We find that the finite-sample distributions can be decisively non-normal (e.g., multimodal). Moreover, we find that a fixed-parameter asymptotic analysis gives highly misleading results. In particular, the 'oracle' property, which is based on a fixed-parameter asymptotic analysis, is shown to not provide a reliable assessment of the estimators' actual performance. For these reasons, we also obtain the asymptotic distributions of the estimators mentioned before in a general 'moving parameter' asymptotic framework, which better captures essential features of the finite-sample distribution. [Interestingly, it turns out that in the consistent model selection case a 'moving parameter' asymptotic framework more general than the usual $n^{-1 / 2}$-local asymptotic framework is necessary to 
exhibit the full range of possible limiting distributions.] Furthermore, we derive the uniform convergence rate of the estimators and show that it is slower than $n^{-1 / 2}$ in the case where the estimators are tuned to perform consistent model selection. This again exposes the misleading character of the 'oracle' property. We also show that the finite-sample distribution of these estimators can not be estimated in any reasonable sense, complementing results of this sort in the literature (Leeb and Pötscher (2006a,b, 2008b), Pötscher (2006)). In a subsequent paper, Pötscher and Schneider (2009), analogous results are obtained for the adaptive LASSO estimator.

We note that penalized maximum likelihood estimators are intimately related to more classical post-model-selection estimators. The distributional properties of the latter estimators have been studied by Sen (1979), Pötscher (1991), and Leeb and Pötscher (2003, 2005, 2006a,b, 2008b).

The paper is organized as follows: The model and the estimators are introduced in Section 2, and the model selection probabilities are discussed in Section 3. Consistency, uniform consistency, and uniform convergence rates of the estimators are the subject of Section 4 The finite-sample distributions are derived in Section 5.1. whereas the asymptotic distributions are studied in Section 5.2. Section 6 provides impossibility results concerning the estimation of the finite-sample distributions of the estimators, and Section 7 concludes and summarizes our main findings. The appendix contains results on the asymptotic distribution in the consistent model selection case when the estimators are scaled by the inverse of the uniform convergence rate obtained in Section 4 rather than by $n^{1 / 2}$.

\section{The Model and the Estimators}

We start with the orthogonal linear regression model

$$
Y=X \beta+u
$$

where $X^{\prime} X$ is diagonal and the vector $u$ is multivariate normal with mean zero and variance covariance matrix $\sigma^{2} I$. The multivariate linear model with orthogonal design occurs in many important settings, including wavelet regression or the analysis of variance. Because we consider penalized least-squares estimators with a penalty term that is separable with respect to $\beta$, the resulting estimators for the components of $\beta$ are mutually independent and each component estimator is equivalent to the corresponding penalized least squares estimator in a univariate Gaussian location model. We therefore restrict attention to this simple model in the sequel without loss of generality.

Suppose $y_{1}, \ldots, y_{n}$ are independent and each distributed as $N\left(\theta, \sigma^{2}\right)$. We assume for simplicity that $\sigma^{2}$ is known, and hence we can set $\sigma^{2}=1$ without loss of generality. Apart from the standard maximum likelihood (least squares) estimator $\bar{y}$ we consider the following estimators:

1. The hard-thresholding estimator $\hat{\theta}_{H}=\bar{y} \mathbf{1}\left(|\bar{y}|>\eta_{n}\right)$ where the threshold $\eta_{n}$ is a positive real number and $\mathbf{1}(\cdot)$ denotes the indicator function. The 
threshold $\eta_{n}$ is a tuning parameter set by the user. The hard-thresholding estimator can be viewed as a penalized least-squares estimator that arises as the solution to the minimization problem ${ }^{1}$

$$
\sum_{t=1}^{n}\left(y_{t}-\theta\right)^{2}+n\left(\eta_{n}^{2}-\left(|\theta|-\eta_{n}\right)^{2} \mathbf{1}\left(|\theta|<\eta_{n}\right)\right)
$$

We also note here that for $\eta_{n}=n^{-1 / 4}$ the hard-thresholding estimator is a simple instance of Hodges' estimator (see, e.g., Lehmann and Casella (1998), pp. 440-443).

2. The soft-thresholding estimator $\hat{\theta}_{S}=\operatorname{sign}(\bar{y})\left(|\bar{y}|-\eta_{n}\right)_{+}$with $\eta_{n}$ as before. [Here $\operatorname{sign}(x)$ is defined as $-1,0$, and 1 in case $x<0, x=0$, and $x>0$, respectively, and $z_{+}$is shorthand for $\max \{z, 0\}$.] That estimator arises as the solution to the penalized least-squares problem

$$
\sum_{t=1}^{n}\left(y_{t}-\theta\right)^{2}+2 n \eta_{n}|\theta|
$$

which shows that $\hat{\theta}_{S}$ coincides with the LASSO in the form considered in Knight and $\mathrm{Fu}$ (2000). Note that the tuning parameter in the latter reference is $\lambda_{n}=2 n \eta_{n}$.

3. The SCAD-estimator of Fan and Li (2001) is - in the present context given by

$$
\hat{\theta}_{S C A D}=\left\{\begin{array}{lll}
\operatorname{sign}(\bar{y})\left(|\bar{y}|-\eta_{n}\right)_{+} & \text {if } \quad|\bar{y}| \leq 2 \eta_{n}, \\
\left\{(a-1) \bar{y}-\operatorname{sign}(\bar{y}) a \eta_{n}\right\} /(a-2) & \text { if } \quad 2 \eta_{n}<|\bar{y}| \leq a \eta_{n} \\
\bar{y} & \text { if } \quad|\bar{y}|>a \eta_{n},
\end{array}\right.
$$

where $a>2$ is an additional tuning parameter. This estimator can be viewed as a simple combination of soft-thresholding for 'small' $|\bar{y}|$ and hard-thresholding for 'large' $|\bar{y}|$, with a (piecewise) linear interpolation inbetween. Alternatively, the estimator can also be obtained as a solution to a penalized least squares problem; see Fan and Li (2001) for details. We note that the SCAD-estimator is closely related to the firm shrinkage estimator of Bruce and Gao (1996).

\section{$3 \quad$ Model Selection Probabilities}

Each of the three estimators discussed above induces a selection between the restricted model $M_{R}$ consisting only of the $N(0,1)$-distribution and the unrestricted model $M_{U}=\{N(\theta, 1): \theta \in \mathbb{R}\}$ in an obvious way, i.e., $M_{R}$ is selected

\footnotetext{
${ }^{1}$ The penalty corresponding to hard thresholding given in Fan and Li (2001) differs from the correct one that we use here, because of a scaling error in equations (2.3) and (2.4) of Fan and $\mathrm{Li}(2001)$.
} 
if the respective estimator for $\theta$ equals zero, and $M_{U}$ is selected otherwise. In the present context, the hard-thresholding estimator $\hat{\theta}_{H}$ is furthermore nothing else than a traditional pre-test estimator that chooses between the unrestricted maximum likelihood estimator $\hat{\theta}_{U}=\bar{y}$ and the restricted maximum likelihood estimator $\hat{\theta}_{R} \equiv 0$ according to the outcome of a $t$-type test for the hypothesis $\theta=0$.

We now study the model selection probabilities, i.e., the probabilities that model $M_{U}$ or $M_{R}$, respectively, is selected. As they add up to one, it suffices to consider one of them. First note that the probability of selecting the model $M_{R}$ is the same for each of the estimators $\hat{\theta}_{H}, \hat{\theta}_{S}$, and $\hat{\theta}_{S C A D}$ (provided the same tuning parameter $\eta_{n}$ is used). This is so because the events $\left\{\hat{\theta}_{H}=0\right\}$, $\left\{\hat{\theta}_{S}=0\right\}$, and $\left\{\hat{\theta}_{S C A D}=0\right\}$ coincide. Hence,

$$
\begin{aligned}
P_{n, \theta}(\hat{\theta}=0) & =P_{n, \theta}\left(|\bar{y}| \leq \eta_{n}\right)=\operatorname{Pr}\left(\left|Z+n^{1 / 2} \theta\right| \leq n^{1 / 2} \eta_{n}\right) \\
& =\Phi\left(n^{1 / 2}\left(-\theta+\eta_{n}\right)\right)-\Phi\left(n^{1 / 2}\left(-\theta-\eta_{n}\right)\right),
\end{aligned}
$$

where $\hat{\theta}$ stands for any of the estimators $\hat{\theta}_{H}, \hat{\theta}_{S}$, and $\hat{\theta}_{S C A D}$, and where $Z$ is a standard normal random variable with cumulative distribution function (cdf) $\Phi$. Here we use $P_{n, \theta}$ to denote the probability governing a sample of size $n$ when $\theta$ is the true parameter, and $\operatorname{Pr}$ to denote a generic probability measure.

In the following we shall always impose the condition that $\eta_{n} \rightarrow 0$ for asymptotic considerations, which guarantees that the probability of incorrectly selecting the restricted model $M_{R}$ (i.e., selecting $M_{R}$ if the true $\theta$ is non-zero) vanishes asymptotically. Conversely, if this probability vanishes asymptotically for every $\theta \neq 0$, then $\eta_{n} \rightarrow 0$ follows. Therefore, the condition $\eta_{n} \rightarrow 0$ is a basic one and without this condition the estimators $\hat{\theta}_{H}, \hat{\theta}_{S}$, and $\hat{\theta}_{S C A D}$ do not seem to be of much interest (from an asymptotic viewpoint). As we shall see in the next section, this basic condition is also equivalent to consistency for $\theta$ of the hard-thresholding (soft-thresholding, SCAD) estimator.

Given the condition $\eta_{n} \rightarrow 0$, two cases need to be distinguished: (i) $n^{1 / 2} \eta_{n} \rightarrow$ $e, 0 \leq e<\infty$ and (ii) $n^{1 / 2} \eta_{n} \rightarrow e=\left.\infty\right|^{2}$ In case (i) the hard-thresholding (softthresholding, SCAD) estimator acts as a conservative model selection procedure, i.e., the probability of selecting the unrestricted model $M_{U}$ has a positive limit even when $\theta=0$, whereas in case (ii) it acts as a consistent model selection procedure, i.e., this probability vanishes in the limit when $\theta=0$. This is immediately seen by inspection of (1). These facts have long been known, see Bauer, Pötscher, and Hackl (1988).

The results discussed in the preceding paragraph are of a 'pointwise' asymptotic nature in the sense that the value of $\theta$ is held fixed when sample size $n$ goes to infinity. As noted before, such pointwise asymptotic results often miss essential aspects of the finite-sample behavior, especially in the context of model selection; cf. Leeb and Pötscher (2005). To obtain large-sample results that better capture finite-sample phenomena, we next present a 'moving parameter'

\footnotetext{
${ }^{2}$ There is no loss in generality here in the sense that the general case where only $\eta_{n} \rightarrow 0$ holds can always be reduced to case (i) or case (ii) by passing to subsequences.
} 
asymptotic analysis, i.e., we allow $\theta$ to vary with $n$ as $n \rightarrow \infty$. The following result shows in particular that convergence of the model selection probability to its limit in a pointwise asymptotic analysis is not uniform in $\theta \in \mathbb{R}$ (in fact, it fails to be uniform in any neighborhood of $\theta=0$ ).

Proposition 1 Let $\hat{\theta}$ be either $\hat{\theta}_{H}, \hat{\theta}_{S}$, or $\hat{\theta}_{S C A D}$. Suppose that $\eta_{n} \rightarrow 0$ and $n^{1 / 2} \eta_{n} \rightarrow e$ with $0 \leq e \leq \infty$.

(i) Assume e $<\infty$ (corresponding to conservative model selection). Suppose the true parameter $\theta_{n} \in \mathbb{R}$ satisfies $n^{1 / 2} \theta_{n} \rightarrow \nu \in \mathbb{R} \cup\{-\infty, \infty\}$. Then

$$
\lim _{n \rightarrow \infty} P_{n, \theta_{n}}(\hat{\theta}=0)=\Phi(-\nu+e)-\Phi(-\nu-e) .
$$

(ii) Assume $e=\infty$ (corresponding to consistent model selection). Suppose $\theta_{n} \in \mathbb{R}$ satisfies $\theta_{n} / \eta_{n} \rightarrow \zeta \in \mathbb{R} \cup\{-\infty, \infty\}$. Then

1. $|\zeta|<1$ implies $\lim _{n \rightarrow \infty} P_{n, \theta_{n}}(\hat{\theta}=0)=1$;

2. $|\zeta|=1$ and $n^{1 / 2}\left(\eta_{n}-\zeta \theta_{n}\right) \rightarrow r$ for some $r \in \mathbb{R} \cup\{-\infty, \infty\}$, implies $\lim _{n \rightarrow \infty} P_{n, \theta_{n}}(\hat{\theta}=0)=\Phi(r)$;

3. $|\zeta|>1$ implies $\lim _{n \rightarrow \infty} P_{n, \theta_{n}}(\hat{\theta}=0)=0$.

Proof. The proof of part (i) is immediate from (1). To prove part (ii) we use (1) to rewrite $P_{n, \theta_{n}}(\hat{\theta}=0)$ as

$$
P_{n, \theta_{n}}(\hat{\theta}=0)=\Phi\left(n^{1 / 2} \eta_{n}\left(1-\theta_{n} / \eta_{n}\right)\right)-\Phi\left(n^{1 / 2} \eta_{n}\left(-1-\theta_{n} / \eta_{n}\right)\right) .
$$

The first and the third claim follow immediately from this. For the second claim, assume first that $\zeta=1$. Then $\Phi\left(n^{1 / 2} \eta_{n}\left(1-\theta_{n} / \eta_{n}\right)\right)=\Phi\left(n^{1 / 2}\left(\eta_{n}-\zeta \theta_{n}\right)\right)$ obviously converges to $\Phi(r)$, whereas $\Phi\left(n^{1 / 2} \eta_{n}\left(-1-\theta_{n} / \eta_{n}\right)\right)$ converges to zero. The case $\zeta=-1$ is handled similarly.

Proposition 1 in fact completely describes the large-sample behavior of the model selection probability without any conditions on the parameter $\theta$, in the sense that all possible accumulation points of the model selection probability along arbitrary sequences of $\theta_{n}$ can be obtained in the following manner: Just apply the result to subsequences and note that, by compactness of $\mathbb{R} \cup\{-\infty, \infty\}$, we can select from each subsequence a further subsequence such that all relevant quantities such as $n^{1 / 2} \theta_{n}, \theta_{n} / \eta_{n}, n^{1 / 2}\left(\eta_{n}-\theta_{n}\right)$, or $n^{1 / 2}\left(\eta_{n}+\theta_{n}\right)$ converge in $\mathbb{R} \cup\{-\infty, \infty\}$ along this further subsequence.

In the conservative model selection case we see from Proposition 1 that the usual local alternative parameter sequences describe the asymptotic behavior. In particular, if $\theta_{n}$ is local to $\theta=0$ in the sense that $\theta_{n}=\nu / n^{1 / 2}$, the local alternatives parameter $\nu$ governs the limiting model selection probability. Deviations of $\theta_{n}$ from $\theta=0$ of order $1 / n^{1 / 2}$ are detected with positive probability asymptotically and deviations of larger order are detected with probability one asymptotically in this case. In the consistent model selection case, however, a different picture emerges. Here, Proposition 1 shows that local deviations of $\theta_{n}$ 
from $\theta=0$ that are of the order $1 / n^{1 / 2}$ are not detected by the model selection procedures at all ${ }^{3}$ In fact, even larger deviations of $\theta$ from zero go asymptotically unnoticed by the model selection procedure, namely as long as $\theta_{n} / \eta_{n} \rightarrow \zeta$, $|\zeta|<1$. [Note that these larger deviations would be picked up by a conservative procedure with probability one asymptotically.] This unpleasant consequence of model selection consistency has a number of repercussions as we shall see later on. For a more detailed discussion of these phenomena in the context of post-model-selection estimators see Leeb and Pötscher (2005).

The speed of convergence of the model selection probability to its limit in part (i) of the proposition is governed by the slower of the convergence speeds of $n^{1 / 2} \eta_{n}$ and $n^{1 / 2} \theta_{n}$. In part (ii) it is exponential in $n^{1 / 2} \eta_{n}$ in cases 1 and 3, and is governed by the convergence speed of $n^{1 / 2} \eta_{n}$ and $n^{1 / 2}\left(\eta_{n}-\zeta \theta_{n}\right)$ in case 2 .

\section{Consistency, uniform consistency, and uni- form convergence rate of $\hat{\theta}_{H}, \hat{\theta}_{S}$, and $\hat{\theta}_{S C A D}$}

It is easy to see that the basic condition $\eta_{n} \rightarrow 0$ discussed in the preceding section is in fact also equivalent to consistency of $\hat{\theta}_{H}$ for $\theta$, i.e., to

$$
\lim _{n \rightarrow \infty} P_{n, \theta}\left(\left|\hat{\theta}_{H}-\theta\right|>\varepsilon\right)=0 \quad \text { for every } \varepsilon>0 \text { and every } \theta \in \mathbb{R} \text {. }
$$

The same is also true for $\hat{\theta}_{S}$ and $\hat{\theta}_{S C A D}$, as is elementary to verify. [At least the sufficiency parts are well-known, see Pötscher (1991) for hard-thresholding, Knight and Fu (2000) for soft-thresholding and Fan and Li (2001) for SCAD.] In fact, under this basic condition on $\eta_{n}$, the estimators are even uniformly consistent with a certain rate as we show next:

Theorem 2 Assume $\eta_{n} \rightarrow 0$. Let $\hat{\theta}$ stand for either $\hat{\theta}_{H}, \hat{\theta}_{S}$, or $\hat{\theta}_{S C A D}$. Then $\hat{\theta}$ is uniformly consistent, i.e.,

$$
\lim _{n \rightarrow \infty} \sup _{\theta \in \mathbb{R}} P_{n, \theta}(|\hat{\theta}-\theta|>\varepsilon)=0 \text { for every } \varepsilon>0 .
$$

In fact, the supremum in the above expression converges to zero exponentially fast for every $\varepsilon>0$. Furthermore, set $a_{n}=\min \left\{n^{1 / 2}, \eta_{n}^{-1}\right\}$. Then for every $\varepsilon>0$ there exists a (nonnegative) real number $M$ such that

$$
\sup _{n \in \mathbb{N}} \sup _{\theta \in \mathbb{R}} P_{n, \theta}\left(a_{n}|\hat{\theta}-\theta|>M\right)<\varepsilon
$$

holds. In particular, $\hat{\theta}$ is uniformly $\min \left\{n^{1 / 2}, \eta_{n}^{-1}\right\}$-consistent.

\footnotetext{
${ }^{3}$ For such deviations this also immediately follows from a contiguity argument.

${ }^{4}$ Knight and $\mathrm{Fu}$ (2000) consider the LASSO-estimator in a linear regression model without an intercept, hence their result does not directly apply to the case considered here.
} 
Proof. We begin with proving uniform consistency of $\hat{\theta}=\hat{\theta}_{H}$. Observe that $\sup _{\theta \in \mathbb{R}} P_{n, \theta}\left(\left|\hat{\theta}_{H}-\theta\right|>\varepsilon\right)$ can be written as

$$
\begin{aligned}
& \sup _{\theta \in \mathbb{R}} P_{n, \theta}\left(\left|(\bar{y}-\theta) \mathbf{1}\left(|\bar{y}|>\eta_{n}\right)-\theta \mathbf{1}\left(|\bar{y}| \leq \eta_{n}\right)\right|>\varepsilon\right) \\
& \leq \sup _{\theta \in \mathbb{R}} P_{n, \theta}\left(|\bar{y}-\theta|>\varepsilon / 2,|\bar{y}|>\eta_{n}\right)+\sup _{\theta \in \mathbb{R}} P_{n, \theta}\left(|\theta|>\varepsilon / 2, \quad|\bar{y}| \leq \eta_{n}\right) \\
& \leq \operatorname{Pr}\left(|Z|>n^{1 / 2} \varepsilon / 2\right)+\sup _{|\theta|>\varepsilon / 2} P_{n, \theta}\left(|\bar{y}| \leq \eta_{n}\right),
\end{aligned}
$$

where $Z$ is standard normally distributed. Now the first term on the far r.h.s. in the above display obviously converges to zero exponentially fast as $n \rightarrow \infty$. In the second term on the far right, the probability gets large as $|\theta|$ gets close to $\varepsilon / 2$. Therefore, the second term on the far r.h.s. equals

$$
\operatorname{Pr}\left(\left|Z+n^{1 / 2} \varepsilon / 2\right| \leq n^{1 / 2} \eta_{n}\right)=\Phi\left(n^{1 / 2}\left(-\varepsilon / 2+\eta_{n}\right)\right)-\Phi\left(n^{1 / 2}\left(-\varepsilon / 2-\eta_{n}\right)\right)
$$

and also goes to zero exponentially fast because $\eta_{n} \rightarrow 0$.

Next, for the soft-thresholding estimator, observe that we have the relation

$$
\hat{\theta}_{S}=\hat{\theta}_{H}-\operatorname{sign}\left(\hat{\theta}_{H}\right) \eta_{n} .
$$

Consequently,

$$
\sup _{\theta \in \mathbb{R}} P_{n, \theta}\left(\left|\hat{\theta}_{H}-\hat{\theta}_{S}\right|>\varepsilon\right) \leq \sup _{\theta \in \mathbb{R}} P_{n, \theta}\left(\eta_{n}>\varepsilon\right)=\mathbf{1}\left(\eta_{n}>\varepsilon\right),
$$

which equals zero for sufficiently large $n$. Hence, the results established so far for $\hat{\theta}_{H}$ carry over to $\hat{\theta}_{S}$.

For the SCAD estimator observe that it is 'sandwiched' between the other two in the sense that

$$
\hat{\theta}_{S} \leq \hat{\theta}_{S C A D} \leq \hat{\theta}_{H}
$$

holds if $\hat{\theta}_{S} \geq 0$, and that the order is reversed if $\hat{\theta}_{S} \leq 0$. This entails the corresponding result for the SCAD estimator.

We next prove uniform $a_{n}$-consistency of $\hat{\theta}_{H}$ : Repeating the arguments from the beginning of the proof with $M / a_{n}$ replacing $\varepsilon$, we see that $\sup _{\theta \in \mathbb{R}} P_{n, \theta}\left(a_{n}\left|\hat{\theta}_{H}-\theta\right|>M\right)$ is bounded from above by

$$
\operatorname{Pr}\left(|Z|>n^{1 / 2} M /\left(2 a_{n}\right)\right)+\operatorname{Pr}\left(\left|Z+n^{1 / 2} M /\left(2 a_{n}\right)\right| \leq n^{1 / 2} \eta_{n}\right) .
$$

Because $n^{1 / 2} / a_{n} \geq 1$, the first term on the right-hand side of the above expression is not larger than $\operatorname{Pr}(|Z|>M / 2)$. The second term equals

$$
\begin{aligned}
& \Phi\left(-n^{1 / 2} M /\left(2 a_{n}\right)+n^{1 / 2} \eta_{n}\right)-\Phi\left(-n^{1 / 2} M /\left(2 a_{n}\right)-n^{1 / 2} \eta_{n}\right) \\
& =\Phi\left(\left(n^{1 / 2} / a_{n}\right)\left(-M / 2+a_{n} \eta_{n}\right)\right)-\Phi\left(\left(n^{1 / 2} / a_{n}\right)\left(-M / 2-a_{n} \eta_{n}\right)\right) .
\end{aligned}
$$


Note that $n^{1 / 2} / a_{n} \geq 1$ and $a_{n} \eta_{n} \leq 1$. For $M>2$, the expression in the above display is therefore not larger than $\Phi(-M / 2+1)$. Uniform $a_{n}$-consistency of $\hat{\theta}_{H}$ follows from this. The proof for $\hat{\theta}_{S}$ and $\hat{\theta}_{S C A D}$ is then similar as before.

For the case where the estimators $\hat{\theta}_{H}, \hat{\theta}_{S}$, and $\hat{\theta}_{S C A D}$ are tuned to perform conservative model selection, the preceding theorem shows that these estimators are uniformly $n^{1 / 2}$-consistent. In contrast, in case the estimators are tuned to perform consistent model selection, the theorem only guarantees uniform $\eta_{n}^{-1}$ consistency; that the estimators do actually not converge faster than $\eta_{n}$ in a uniform sense in this case will be shown in Section 5.2.2.

Remark 3 Let $\hat{\theta}$ denote any one of the estimators $\hat{\theta}_{H}, \hat{\theta}_{S}$, or $\hat{\theta}_{S C A D}$. In case $n^{1 / 2} \eta_{n} \rightarrow e=0$ it is easy to see that $\hat{\theta}$ is uniformly asymptotically equivalent to $\hat{\theta}_{U}=\bar{y}$ in the sense that $\lim _{n \rightarrow \infty} \sup _{\theta \in \mathbb{R}} P_{n, \theta}\left(n^{1 / 2}|\hat{\theta}-\bar{y}|>\varepsilon\right)=0$ for every $\varepsilon>0$. [For $\hat{\theta}=\hat{\theta}_{H}$, this follows easily from Proposition 1] for $\hat{\theta}=\hat{\theta}_{S}$ it follows then from (2), and for $\hat{\theta}=\hat{\theta}_{S C A D}$ from (3).]

\section{The distributions of $\hat{\theta}_{H}, \hat{\theta}_{S}$, and $\hat{\theta}_{S C A D}$}

\subsection{Finite-sample distributions}

For purpose of comparison we note the obvious fact that the distribution of the unrestricted maximum likelihood estimator $\hat{\theta}_{U}=\bar{y}$ (corresponding to model $\left.M_{U}\right)$ as well as the distribution of the restricted maximum likelihood estimator $\hat{\theta}_{R} \equiv 0$ (corresponding to model $\left.M_{R}\right)$ are normal; more precisely, $n^{1 / 2}\left(\hat{\theta}_{U}-\theta\right)$ is $N(0,1)$-distributed and $n^{1 / 2}\left(\hat{\theta}_{R}-\theta\right)$ is $N\left(-n^{1 / 2} \theta, 0\right)$-distributed, where the singular normal distribution is to be interpreted as pointmass at $-n^{1 / 2} \theta$. For the hard-thresholding estimator, the finite-sample distribution $F_{H, n, \theta}$ of $n^{1 / 2}\left(\hat{\theta}_{H}-\theta\right)$ is of the form

$$
\begin{aligned}
d F_{H, n, \theta}(x)=\{ & \left.\Phi\left(n^{1 / 2}\left(-\theta+\eta_{n}\right)\right)-\Phi\left(n^{1 / 2}\left(-\theta-\eta_{n}\right)\right)\right\} d \delta_{-n^{1 / 2} \theta}(x) \\
& +\phi(x) \mathbf{1}\left(\left|x+n^{1 / 2} \theta\right|>n^{1 / 2} \eta_{n}\right) d x
\end{aligned}
$$

where $\delta_{z}$ denotes pointmass at $z$ and $\phi$ denotes the standard normal density. Relation (4) is most easily obtained by writing $P_{n, \theta}\left(n^{1 / 2}\left(\hat{\theta}_{H}-\theta\right) \leq x\right)$ as the sum of $P_{n, \theta}\left(n^{1 / 2}\left(\hat{\theta}_{H}-\theta\right) \leq x, \hat{\theta}_{H}=0\right)$ and $P_{n, \theta}\left(n^{1 / 2}\left(\hat{\theta}_{H}-\theta\right) \leq x, \hat{\theta}_{H} \neq 0\right)$. This also shows that the two terms in (4) correspond to the distribution of $n^{1 / 2}\left(\hat{\theta}_{H}-\theta\right)$ conditional on the events $\left\{\hat{\theta}_{H}=0\right\}$ and $\left\{\hat{\theta}_{H} \neq 0\right\}$, respectively, multiplied by the probability of the respective events. Relation (4) also follows as a special case of Leeb and Pötscher (2003), which provides the finite-sample as well as the asymptotic distributions of a general class of post-model-selection estimators. We recognize that the distribution of the hard-thresholding estimator is a mixture of two components: The first one is a singular normal distribution (i.e., pointmass) and coincides with the distribution of the restricted maximum likelihood estimator. The second one is absolutely continuous and represents an 
'excised' version of the normal distribution of the unrestricted maximum likelihood estimator. Note that the absolutely continuous part in (4) is bimodal and hence is distinctly non-normal. The shape of the distribution of $n^{1 / 2}\left(\hat{\theta}_{H}-\theta\right)$ is exemplified in Figure 1.

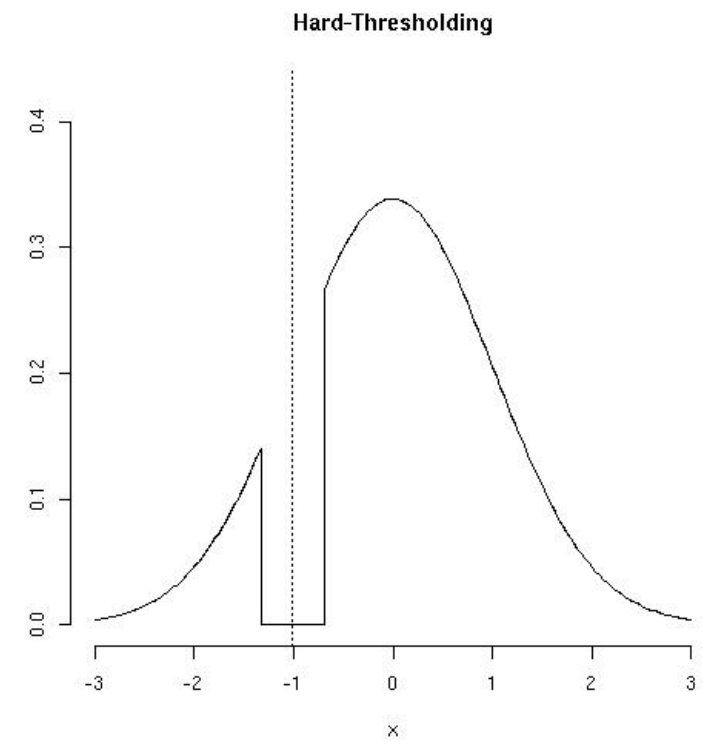

Figure 1: Distribution of $n^{1 / 2}\left(\hat{\theta}_{H}-\theta\right)$ for $n=40, \theta=0.16$, and $\eta_{n}=0.05$. The density of the absolutely continuous part is shown by the solid curve, which is discontinuous at $x=n^{1 / 2}\left(-\theta-\eta_{n}\right)$ and $x=$ $n^{1 / 2}\left(-\theta+\eta_{n}\right)$. [For better readability, the left- and right-hand limits at discontinuity points are joined by line segments.] The vertical dotted line indicates the location of the point-mass at $-n^{1 / 2} \theta$; the weight of the point-mass, i.e., the multiplier of $d \delta_{-n^{1 / 2} \theta}(x)$ in (4), equals 0.15 . For other values of the constants involved here, a similar picture is obtained.

The finite-sample distribution $F_{S, n, \theta}$ of $n^{1 / 2}\left(\hat{\theta}_{S}-\theta\right)$ is given by

$$
\begin{aligned}
d F_{S, n, \theta}(x)= & \left\{\Phi\left(n^{1 / 2}\left(-\theta+\eta_{n}\right)\right)-\Phi\left(n^{1 / 2}\left(-\theta-\eta_{n}\right)\right)\right\} d \delta_{-n^{1 / 2} \theta}(x) \\
& +\phi\left(x-n^{1 / 2} \eta_{n}\right) \mathbf{1}\left(x+n^{1 / 2} \theta<0\right) d x \\
& +\phi\left(x+n^{1 / 2} \eta_{n}\right) \mathbf{1}\left(x+n^{1 / 2} \theta>0\right) d x .
\end{aligned}
$$

For later use we note that this implies

$$
\left.\left.F_{S, n, \theta}(x)=\Phi\left(x+n^{1 / 2} \eta_{n}\right)\right) \mathbf{1}\left(x \geq-n^{1 / 2} \theta\right)+\Phi\left(x-n^{1 / 2} \eta_{n}\right)\right) \mathbf{1}\left(x<-n^{1 / 2} \theta\right) .
$$


Relation (5) is obtained from a derivation similar to that of (4), namely by representing $P_{n, \theta}\left(n^{1 / 2}\left(\hat{\theta}_{S}-\theta\right) \leq x\right)$ as the sum of $P_{n, \theta}\left(n^{1 / 2}\left(\hat{\theta}_{S}-\theta\right) \leq x, \hat{\theta}_{S}=0\right)$, $P_{n, \theta}\left(n^{1 / 2}\left(\hat{\theta}_{S}-\theta\right) \leq x, \hat{\theta}_{S}>0\right)$, and $P_{n, \theta}\left(n^{1 / 2}\left(\hat{\theta}_{S}-\theta\right) \leq x, \hat{\theta}_{S}<0\right)$. Similar to before, the three terms in $(5)$ correspond to the distributions of $n^{1 / 2}\left(\hat{\theta}_{S}-\theta\right)$ conditional on the events $\left\{\hat{\theta}_{S}=0\right\},\left\{\hat{\theta}_{S}>0\right\}$, and $\left\{\hat{\theta}_{S}<0\right\}$, respectively, multiplied by the respective probabilities of these events. The distribution in (5) is again a mixture of a singular normal distribution and of an absolutely continuous part, which is now the sum of two normal densities, each with a truncated tail. Figure 2 exemplifies a typical shape of this distribution.

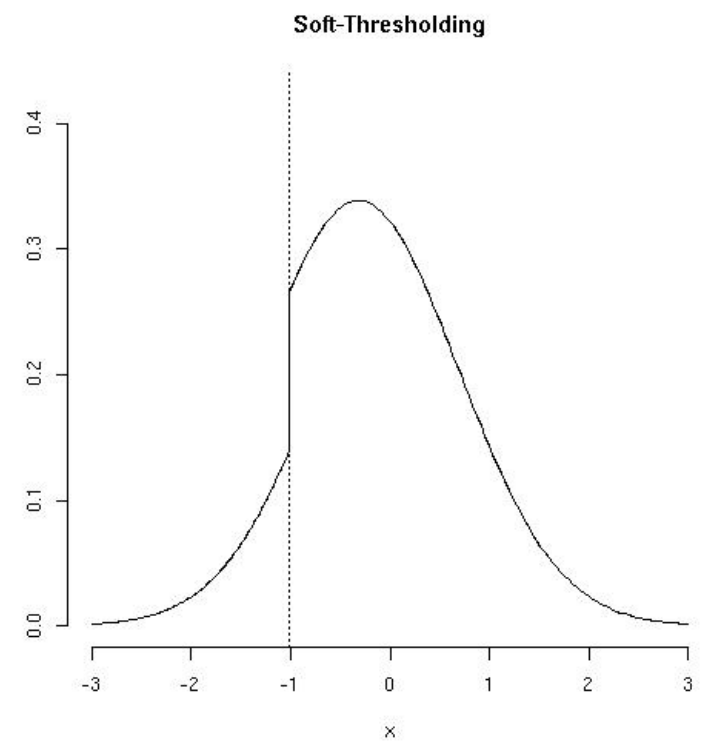

Figure 2: Distribution of $n^{1 / 2}\left(\hat{\theta}_{S}-\theta\right)$. The choice of constants and the interpretation of the image is the same as in Figure 1.

The finite-sample distribution of the SCAD-estimator is obtained in a similar vein: Decomposing the probability $P_{n, \theta}\left(n^{1 / 2}\left(\hat{\theta}_{S C A D}-\theta\right) \leq x\right)$ into a sum of seven terms by decomposing the relevant event into its intersection with the events $\left\{|\bar{y}| \leq \eta_{n}\right\},\left\{\eta_{n}<\bar{y} \leq 2 \eta_{n}\right\},\left\{2 \eta_{n}<\bar{y} \leq a \eta_{n}\right\},\left\{a \eta_{n}<\bar{y}\right\},\left\{-2 \eta_{n} \leq\right.$ $\left.\bar{y}<-\eta_{n}\right\},\left\{-a \eta_{n} \leq \bar{y}<-2 \eta_{n}\right\}$, and $\left\{\bar{y}<-a \eta_{n}\right\}$, shows that the distribution $F_{S C A D, n, \theta}$ of $n^{1 / 2}\left(\hat{\theta}_{S C A D}-\theta\right)$ is of the form

$$
\begin{array}{r}
d F_{S C A D, n, \theta}(x)=\left\{\Phi\left(n^{1 / 2}\left(-\theta+\eta_{n}\right)\right)-\Phi\left(n^{1 / 2}\left(-\theta-\eta_{n}\right)\right)\right\} d \delta_{-n^{1 / 2} \theta}(x) \\
+\left\{f_{1}(x)+f_{2}(x)+f_{3}(x)+f_{-1}(x)+f_{-2}(x)+f_{-3}(x)\right\} d x,
\end{array}
$$


where

$$
\begin{aligned}
f_{1}(x)= & \phi\left(x+n^{1 / 2} \eta_{n}\right) \mathbf{1}\left(0<x+n^{1 / 2} \theta \leq n^{1 / 2} \eta_{n}\right), \\
f_{2}(x)= & \frac{a-2}{a-1} \phi\left(\left\{(a-2) x-n^{1 / 2} \theta+a n^{1 / 2} \eta_{n}\right\} /(a-1)\right) \times \\
& \mathbf{1}\left(n^{1 / 2} \eta_{n}<x+n^{1 / 2} \theta \leq a n^{1 / 2} \eta_{n}\right), \\
f_{3}(x)= & \phi(x) \mathbf{1}\left(x+n^{1 / 2} \theta>n^{1 / 2} a \eta_{n}\right),
\end{aligned}
$$

and where $f_{-1}(x), f_{-2}(x)$, and $f_{-3}(x)$ are defined as $f_{1}(x), f_{2}(x)$, and $f_{3}(x)$, respectively, but with $-x$ replacing $x$ and with $-\theta$ replacing $\theta$ in the formulae. Like in the case of the other estimators, the distribution of the SCAD-estimator is a mixture of a singular normal distribution and an absolutely continuous part, the latter being more complicated here as it is the sum of six pieces, each obtained from normal distributions by truncation or excision. As shown in Figure 3, the absolutely continuous part of $F_{S C A D, n, \theta}$ can be multimodal.

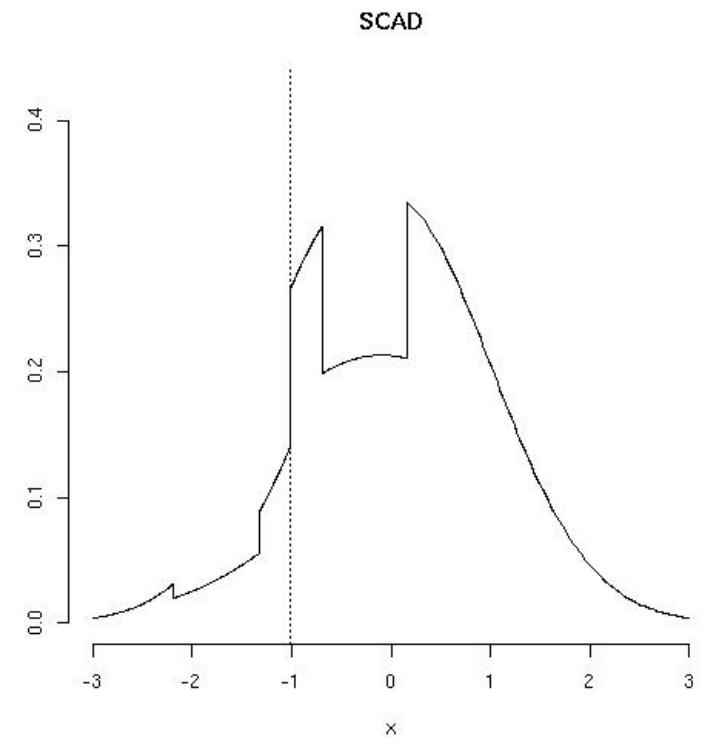

Figure 3: Distribution of $n^{1 / 2}\left(\hat{\theta}_{S C A D}-\theta\right)$. The tuning-parameter $a$ is chosen as $a=3.7$ here, cf. Fan and Li (2001); the choice of the other constants and the interpretation of the image is the same as in Figure 1. The graph for the SCAD estimator coincides with that for the soft-thresholding estimator inside a neighborhood of the location of the atomic part at $-n^{1 / 2} \theta$ (vertical dotted line), and with that for the hard-thresholding estimator outside of a (larger) neighborhood of $-n^{1 / 2} \theta$. The area between these two regions corresponds to the dips shown in the figure. 
In summary, we see that the finite-sample distributions of the estimators $\hat{\theta}_{H}$, $\hat{\theta}_{S}$, and $\hat{\theta}_{S C A D}$ are typically highly non-normal and can be multimodal. As a point of interest, we also note that the computations leading to the above formulae also deliver the conditional finite-sample distributions of the estimators $\hat{\theta}_{H}, \hat{\theta}_{S}$, and $\hat{\theta}_{S C A D}$, respectively, conditional on selecting model $M_{R}$ or $M_{U}$. In particular, we note that the conditional distribution of each of these estimators, conditional on having selected the restricted model $M_{R}$, coincides with the distribution of the restricted maximum likelihood estimator $\hat{\theta}_{R}$; in contrast, conditional on selecting the unrestricted model $M_{U}$, the conditional distribution is not identical to the distribution of the unrestricted maximum likelihood estimator $\hat{\theta}_{U}$, but is more complicated. This phenomenon applies also to large classes of post-model-selection estimators; see Pötscher (1991) and Leeb and Pötscher (2003) for more discussion.

\section{$5.2 \quad$ Asymptotic distributions}

We next obtain the asymptotic distributions of $\hat{\theta}_{H}, \hat{\theta}_{S}$, and $\hat{\theta}_{S C A D}$. We present the asymptotic distributional results under general 'moving parameter' asymptotics, where the true parameter $\theta_{n}$ can depend on sample size, because considering only fixed-parameter asymptotics may paint a quite misleading picture of the behavior of the estimators (cf. Leeb and Pötscher (2003, 2005)). In fact, the results given below amount to a complete description of all possible accumulation points of the finite-sample distributions of the estimators in question, cf. Remarks 8 and 12. Not surprisingly, the results in the conservative model selection case are different from the ones in the consistent model selection case.

\subsubsection{Conservative case}

Here we characterize the large-sample behavior of the distributions of $\hat{\theta}_{H}, \hat{\theta}_{S}$, and $\hat{\theta}_{S C A D}$ for the case where these estimators are tuned to perform conservative model selection.

Theorem 4 Consider the hard-thresholding estimator with $\eta_{n} \rightarrow 0$ and $n^{1 / 2} \eta_{n} \rightarrow e, 0 \leq e<\infty$. Suppose the true parameter $\theta_{n} \in \mathbb{R}$ satisfies $n^{1 / 2} \theta_{n} \rightarrow \nu \in \mathbb{R} \cup\{-\infty, \infty\}$. Then $F_{H, n, \theta_{n}}$ converges weakly to the distribution given by

$$
\{\Phi(-\nu+e)-\Phi(-\nu-e)\} d \delta_{-\nu}(x)+\phi(x) \mathbf{1}(|x+\nu|>e) d x .
$$

[Note that (8) reduces to a standard normal distribution in case $|\nu|=\infty$ or $e=0$.]

Proof. 5 Recall that the finite-sample distribution is given in (4). Convergence of the weights $\Phi\left(n^{1 / 2}\left(-\theta+\eta_{n}\right)\right)-\Phi\left(n^{1 / 2}\left(-\theta-\eta_{n}\right)\right)$ to $\Phi(-\nu+e)-\Phi(-\nu-e)$

\footnotetext{
${ }^{5}$ Theorem 4 is essentially a special case of results obtained in Leeb and Pötscher (2003) for a more general class of post-model-selection estimators. The proof of this result is included here because of its brevity and illustrative value.
} 
is obvious (cf. proof of Proposition 1). Hence, the atomic part of $F_{H, n, \theta_{n}}$ converges weakly to the atomic part of $(8)$ if $|\nu|<\infty$ and $e>0$; if $|\nu|=\infty$ or if $e=0$, the total mass of the atomic part converges to zero. The density of the absolutely continuous part of $F_{H, n, \theta_{n}}$ is easily seen to converge Lebesgue almost everywhere (in fact everywhere on $\mathbb{R}$ except possibly at $x=-\nu \pm e$ ) to the density of the absolutely continuous part of (8). Also the total mass of the absolutely continuous part is seen to converge to the total mass of the absolutely continuous part of (8). By an application of Scheffé's Lemma, the densities converge in absolute mean, and hence the absolutely continuous part converges in the total variation sense.

The fixed-parameter asymptotic distribution is obtained from Theorem 4 by letting $\theta_{n} \equiv \theta$ : If $\theta=0$, the pointwise asymptotic distribution of the hardthresholding estimator is seen to be

$$
\{\Phi(e)-\Phi(-e)\} d \delta_{0}(x)+\phi(x) \mathbf{1}(|x|>e) d x
$$

which coincides with the finite-sample distribution (4) in this case except for replacing $n^{1 / 2} \eta_{n}$ by its limiting value $e$. However, if $\theta \neq 0$, the pointwise asymptotic distribution is always standard normal, which clearly misrepresents the actual distribution (4). This disagreement is most pronounced in the statistically interesting case where $\theta$ is close to, but not equal to, zero (e.g., $\theta \sim n^{-1 / 2}$ ). In contrast, the distribution (8) much better captures the behavior of the finitesample distribution also in this case because (8) coincides with (4) except for the fact that $n^{1 / 2} \eta_{n}$ and $n^{1 / 2} \theta_{n}$ have settled down to their limiting values.

Theorem 5 Consider the soft-thresholding estimator with $\eta_{n} \rightarrow 0$ and $n^{1 / 2} \eta_{n} \rightarrow e, 0 \leq e<\infty$. Suppose the true parameter $\theta_{n} \in \mathbb{R}$ satisfies $n^{1 / 2} \theta_{n} \rightarrow \nu \in \mathbb{R} \cup\{-\infty, \infty\}$. Then $F_{S, n, \theta_{n}}$ converges weakly to the distribution given by

$$
\begin{aligned}
& \{\Phi(-\nu+e)-\Phi(-\nu-e)\} d \delta_{-\nu}(x) \\
& +\{\phi(x+e) \mathbf{1}(x>-\nu)+\phi(x-e) \mathbf{1}(x<-\nu)\} d x .
\end{aligned}
$$

[Note that (9) reduces to a $N(-\operatorname{sign}(\nu) e, 1)$-distribution in case $|\nu|=\infty$ or $e=0$.]

The proof is completely analogous to the proof of Theorem 4. Since softthresholding arises as a special case of the LASSO-estimator, the above result is closely related to the results in Knight and $\mathrm{Fu}(2000){ }^{6}$ Similar to the case of hard-thresholding, a fixed-parameter asymptotic analysis only partially reflects the finite-sample behavior of the estimator: In case $\theta=0$, the pointwise asymptotic distribution is

$$
\{\Phi(e)-\Phi(-e)\} d \delta_{0}(x)+\{\phi(x-e) \mathbf{1}(x<0)+\phi(x+e) \mathbf{1}(x>0)\} d x .
$$

\footnotetext{
${ }^{6}$ Since Knight and Fu (2000) consider the LASSO-estimator in a linear regression model without an intercept, their results do not directly apply to the model considered here. However, their results can easily be modified to also cover linear regression with an intercept.
} 
However, if $\theta \neq 0$, the pointwise limit distribution is $N(-\operatorname{sign}(\theta) e, 1)$, which is not in good agreement with the finite-sample distribution (5), especially in the statistically interesting case where $\theta$ is close to, but not equal to, zero (e.g., $\left.\theta \sim n^{-1 / 2}\right)$. In contrast, (9) is in better agreement with (5) also in this case in the sense that (9) coincides with (5), except that $n^{1 / 2} \eta_{n}$ and $n^{1 / 2} \theta_{n}$ have settled down to their limiting values.

Theorem 6 Consider the SCAD estimator with $\eta_{n} \rightarrow 0$ and $n^{1 / 2} \eta_{n} \rightarrow e, 0 \leq$ $e<\infty$. Suppose the true parameter $\theta_{n} \in \mathbb{R}$ satisfies $n^{1 / 2} \theta_{n} \rightarrow \nu \in \mathbb{R} \cup\{-\infty, \infty\}$. Then $F_{S C A D, n, \theta_{n}}$ converges weakly to the distribution given by

$$
\begin{aligned}
& \{\Phi(-\nu+e)-\Phi(-\nu-e)\} d \delta_{-\nu}(x) \\
& +\quad\{\phi(x+e) \mathbf{1}(0<x+\nu \leq e)+\phi(x-e) \mathbf{1}(-e \leq x+\nu<0) \\
& \quad+\frac{a-2}{a-1} \phi(\{(a-2) x-\nu+a e\} /(a-1)) \mathbf{1}(e<x+\nu \leq a e) \\
& \quad+\frac{a-2}{a-1} \phi(\{(a-2) x-\nu-a e\} /(a-1)) \mathbf{1}(-a e \leq x+\nu<-e) \\
& \quad+\phi(x) \mathbf{1}(|x+\nu|>a e)\} d x .
\end{aligned}
$$

[Note that 110$)$ reduces to a standard normal distribution in case $|\nu|=\infty$ or $e=0$.]

The proof of Theorem 6 is again completely analogous to that of Theorem 4. As with the hard- and soft-thresholding estimators discussed before, a fixedparameter asymptotic analysis of the SCAD estimator only partially reflects its finite-sample behavior: In case $\theta=0$, the pointwise asymptotic distribution is given by 110 with $\nu=0$, but in case $\theta \neq 0$ it is given by $N(0,1)$, which is definitely not in good agreement with the finite-sample distribution (7), especially in the statistically interesting case where $\theta$ is different from, but close to, zero, e.g., $\theta \sim n^{-1 / 2}$. In contrast, 10 is in much better agreement with 7 in view of the fact that (10) coincides with (7), except that $n^{1 / 2} \eta_{n}$ and $n^{1 / 2} \theta_{n}$ have settled down to their limiting values.

We note that the mathematical reason for the failure of the pointwise asymptotic distribution to capture the behavior of the finite-sample distribution well is that the convergence of the latter to the former is not uniform in the underlying parameter $\theta$. See Leeb and Pötscher $(2003,2005)$ for more discussion in the context of post-model-selection estimators.

Remark 7 If $|\nu|=\infty$, or $e=0$, or $n^{1 / 2} \theta_{n}=\nu$ does not depend on $n$, the convergence in the above three theorems is even in the total variation distance. In the first two cases this follows because the total mass of the atomic part converges to zero; in the third case it follows because the location of the pointmass is independent of $n$.

Remark 8 The above theorems actually completely describe the limiting behavior of the finite-sample distributions of $\hat{\theta}_{H}, \hat{\theta}_{S}$, and $\hat{\theta}_{S C A D}$ without any 
condition on the sequence of parameters $\theta_{n}$. To see this, just apply the theorems to subsequences and note that by compactness of $\mathbb{R} \cup\{-\infty, \infty\}$ we can select from every subsequence a further subsequence such that $n^{1 / 2} \theta_{n}$ converges in $\mathbb{R} \cup\{-\infty, \infty\}$ along this further subsequence.

\subsubsection{Consistent case}

In the case where the estimators $\hat{\theta}_{H}, \hat{\theta}_{S}$, and $\hat{\theta}_{S C A D}$ are tuned to perform consistent model selection (i.e., $\eta_{n} \rightarrow 0$ and $n^{1 / 2} \eta_{n} \rightarrow \infty$ ), the fixed-parameter limiting behavior of the finite-sample distributions is particularly simple: The finite-sample distribution of the hard-thresholding estimator converges to the $N(0,0)$-distribution (i.e., to pointmass at 0 ) if $\theta=0$, and to the $N(0,1)$ distribution if $\theta \neq 0$; cf. Lemma 1 in Pötscher (1991). In other words, the pointwise asymptotic distribution of $n^{1 / 2}\left(\hat{\theta}_{H}-\theta\right)$ coincides with the asymptotic distribution of the restricted maximum likelihood estimator if $\theta=0$, and coincides with the asymptotic distribution of the unrestricted maximum likelihood estimator if $\theta \neq 0$. The hard-thresholding estimator, when tuned in this way, therefore satisfies what has sometimes been dubbed the 'oracle' property in the literature 7 The SCAD-estimator with the same tuning is also known to possess the 'oracle' property; cf. Fan and Li (2001). With the same tuning, the softthresholding has a somewhat different pointwise asymptotic behavior which is discussed later.

The 'oracle' property of the hard-thresholding estimator and the SCADestimator implies in particular that both estimators are $n^{1 / 2}$-consistent. In Theorem 2, however, we have - in contrast to the conservative model selection case - only been able to establish uniform $\eta_{n}^{-1}$-consistency and not uniform $n^{1 / 2}$-consistency. This begs the question whether Theorem 2 is just not sharp enough or whether the estimators actually are not uniformly $n^{1 / 2}$-consistent. It furthermore raises the question of the behavior of the finite-sample distributions of $n^{1 / 2}\left(\hat{\theta}_{H}-\theta\right), n^{1 / 2}\left(\hat{\theta}_{S}-\theta\right)$, and $n^{1 / 2}\left(\hat{\theta}_{S C A D}-\theta\right)$ in a 'uniform' asymptotic framework. The three results that follow answer this by determining the limits of the finite-sample distributions of $\hat{\theta}_{H}, \hat{\theta}_{S}$, and $\hat{\theta}_{S C A D}$ under general 'moving parameter' asymptotics when the estimators are tuned to perform consistent model selection.

Theorem 9 Consider the hard-thresholding estimator with $\eta_{n} \rightarrow 0$ and $n^{1 / 2} \eta_{n} \rightarrow \infty$. Assume that $\theta_{n} / \eta_{n} \rightarrow \zeta$ for some $\zeta \in \mathbb{R} \cup\{-\infty, \infty\}$ and that $n^{1 / 2} \theta_{n} \rightarrow \nu$ for some $\nu \in \mathbb{R} \cup\{-\infty, \infty\}$. [Note that in case $\zeta \neq 0$ the convergence of $n^{1 / 2} \theta_{n}$ already follows from that of $\theta_{n} / \eta_{n}$, and $\nu$ is then given by $\nu=\operatorname{sign}(\zeta) \infty$.]

1. If $|\zeta|<1$, then $F_{H, n, \theta_{n}}$ approaches pointmass at $-\nu$. In case $|\nu|<\infty$, this means that $F_{H, n, \theta_{n}}$ converges weakly to pointmass at $-\nu$; in case $|\nu|=\infty$,

${ }^{7}$ This does not come as a surprise, since post-model-selection estimators based on a consistent model selection procedure in general satisfy the 'oracle' property as already noted in Lemma 1 of Pötscher (1991); but see also the warning issued in the discussion following that lemma. 
this means that the total mass of $F_{H, n, \theta_{n}}$ escapes to $-\nu$, in the sense that $F_{H, n, \theta_{n}}(x) \rightarrow 0$ for every $x \in \mathbb{R}$ if $-\nu=\infty$, and $F_{H, n, \theta_{n}}(x) \rightarrow 1$ for every $x \in \mathbb{R}$ if $-\nu=-\infty$.

2. If $|\zeta|=1$ and $n^{1 / 2}\left(\eta_{n}-\zeta \theta_{n}\right) \rightarrow r$ for some $r \in \mathbb{R} \cup\{-\infty, \infty\}$, then $F_{H, n, \theta_{n}}(x)$ converges to

$$
\Phi(r) \mathbf{1}(\zeta=1)+\int_{-\infty}^{x} \phi(u) \mathbf{1}(\zeta u>r) d u
$$

for every $x \in \mathbb{R}$. This limit corresponds to pointmass at $-\nu=\operatorname{sign}(-\zeta) \infty$ if $r=\infty$, and otherwise represents a convex combination of pointmass at $-\nu=\operatorname{sign}(-\zeta) \infty$ and an absolutely continuous distribution whose density, a kind of truncated standard normal, is given by $(1-\Phi(r))^{-1}$ times the integrand in the above formula; the weights in that convex combination are given by $\Phi(r)$ and $(1-\Phi(r))$, respectively. [The weight of the absolutely continuous component equals one in case $r=-\infty$; in this case, convergence is in fact in total variation distance.]

3. If $1<|\zeta| \leq \infty$, then $F_{H, n, \theta_{n}}$ converges weakly to $\Phi$, the standard normal cdf. [In fact, convergence is in total variation distance.]

Proof. Proposition 1 shows that the total mass of the atomic part of $F_{H, n, \theta_{n}}$ converges to one under the conditions of part 1 . Because the atomic part is located at $-n^{1 / 2} \theta_{n}$ in view of (4), part 1 follows immediately.

For part 2, assume first that $\zeta=1$. Proposition 1 shows that the total mass of the atomic part of $F_{H, n, \theta_{n}}$ converges to $\Phi(r)$. Furthermore, $n^{1 / 2} \theta_{n} \rightarrow \infty$ certainly holds, which implies that the atomic part escapes to $-\infty$. If $r=\infty$, we are hence done. Suppose now that $r<\infty$. In (4), the boundaries of the 'excision interval' of the absolutely continuous part of $F_{H, n, \theta_{n}}$, i.e., $-n^{1 / 2}\left(\eta_{n}+\theta_{n}\right)=$ $-n^{1 / 2} \eta_{n}\left(1+\theta_{n} / \eta_{n}\right)$ and $n^{1 / 2}\left(\eta_{n}-\theta_{n}\right)$ then converge to $-\infty$ and $r$, respectively. This shows that

$$
\phi(x) \mathbf{1}\left(\left|x+n^{1 / 2} \theta_{n}\right|>n^{1 / 2} \eta_{n}\right) \quad \rightarrow \quad \phi(x) \mathbf{1}(x>r)
$$

for Lebesgue almost every $x \in \mathbb{R}$. The Dominated Convergence Theorem then shows that the convergence in the above display also holds in absolute mean. This completes the proof of part 2 in case $\zeta=1$. The case where $\zeta=-1$ is treated similarly.

Under the conditions of part 3, Proposition 1 shows that the total mass of the absolutely continuous part converges to one. Furthermore, the boundaries of the 'excision interval' in (4), i.e., $-n^{1 / 2}\left(\eta_{n}+\theta_{n}\right)=-n^{1 / 2} \eta_{n}\left(1+\theta_{n} / \eta_{n}\right)$ and $n^{1 / 2}\left(\eta_{n}-\theta_{n}\right)=n^{1 / 2} \eta_{n}\left(1-\theta_{n} / \eta_{n}\right)$, diverge either both to $\infty$ or both to $-\infty$, because $|\zeta|>1$. This implies that

$$
\phi(x) \mathbf{1}\left(\left|x+n^{1 / 2} \theta_{n}\right|>n^{1 / 2} \eta_{n}\right) \quad \rightarrow \quad \phi(x)
$$

for every $x \in \mathbb{R}$. Together with the Dominated Convergence Theorem this completes the proof. 
The fixed-parameter asymptotic behavior of the hard-thresholding estimator discussed earlier, including the 'oracle' property, can clearly be recovered from the above theorem by setting $\theta_{n} \equiv \theta$. However, the theorem shows that the asymptotic behavior of the hard-thresholding estimator is more complicated than what the 'oracle' property predicts. In particular, the theorem shows that the hard-thresholding estimator is not uniformly $n^{1 / 2}$-consistent as the sequence of finite-sample distributions is not stochastically bounded in all cases. [In that sense scaling by $n^{1 / 2}$ does not appear to be the natural thing to do, see the discussion below as well as the appendix.] Furthermore, as shown by (4), the finite-sample distribution is highly non-normal, whereas the pointwise asymptotic distribution is always normal and thus can not capture essential features of the finite-sample distribution. In contrast, the asymptotic distribution given in Theorem 9 is also non-normal in some cases. All this goes to show that the 'oracle' property, which is based on the pointwise asymptotic distribution only, paints a highly misleading picture of the behavior of the hard-thresholding estimator and should not be taken at face value 8 A result for a certain postmodel-selection estimator that is related to Theorem 9 above can be found in Appendix A of Leeb and Pötscher (2005).

Theorem 10 Consider the soft-thresholding estimator with $\eta_{n} \rightarrow 0$ and $n^{1 / 2} \eta_{n} \rightarrow \infty$. Assume that $n^{1 / 2} \theta_{n} \rightarrow \nu \in \mathbb{R} \cup\{-\infty, \infty\}$. Then $F_{S, n, \theta_{n}}$ approaches pointmass at $-\nu$. In case $|\nu|<\infty$, this means that $F_{S, n, \theta_{n}}$ converges weakly to pointmass at $-\nu$; in case $|\nu|=\infty$, it means that the total mass of $F_{S, n, \theta_{n}}$ escapes to $-\nu$, in the sense that $F_{S, n, \theta_{n}}(x) \rightarrow 0$ for every $x \in \mathbb{R}$ if $-\nu=\infty$, and $F_{S, n, \theta_{n}}(x) \rightarrow 1$ for every $x \in \mathbb{R}$ if $-\nu=-\infty$.

Proof. From (6) we have that $F_{S, n, \theta_{n}}(x)=\Phi\left(x+n^{1 / 2} \eta_{n}\right)$ for $x>-n^{1 / 2} \theta_{n}$ and $F_{S, n, \theta_{n}}(x)=\Phi\left(x-n^{1 / 2} \eta_{n}\right)$ for $x<-n^{1 / 2} \theta_{n}$. Because $n^{1 / 2} \eta_{n} \rightarrow \infty$, this entails that $F_{S, n, \theta_{n}}(x)$ converges to one for each $x>-\nu$ and to zero for each $x<-\nu$.

The fixed-parameter asymptotic distribution of the soft-thresholding estimator is obtained by setting $\theta_{n} \equiv \theta$ in the above theorem: It is $N(0,0)$ (i.e., pointmass at 0 ) if $\theta=0$; if $\theta \neq 0$ the total mass of the finite-sample distribution escapes to $\operatorname{sign}(-\theta) \infty$. Hence, the soft-thresholding estimator when tuned to act as a consistent model selector is not even pointwise $n^{1 / 2}$-consistent (Zou (2006)) and certainly does not satisfy the 'oracle' property. [This contradicts an incorrect claim in Zhao and $\mathrm{Yu}$ (2006, Section 2.1) to the effect that tuning LASSO to act as a consistent model selector results in an asymptotically normal estimator.] The fact that this estimator is not pointwise $n^{1 / 2}$-consistent also suggest studying the asymptotic distribution under a scaling that increases slower than $n^{1 / 2}$, an issue that we take up further below; cf. also the appendix.

Theorem 11 Consider the SCAD estimator with $\eta_{n} \rightarrow 0$ and $n^{1 / 2} \eta_{n} \rightarrow \infty$. Assume that $\theta_{n} / \eta_{n} \rightarrow \zeta$ for some $\zeta \in \mathbb{R} \cup\{-\infty, \infty\}$ and that $n^{1 / 2} \theta_{n} \rightarrow \nu$ for

${ }^{8}$ This is of course not new and has been observed more than 50 years ago in the context of Hodges' estimator. For more discussion of the problematic nature of the 'oracle' property see Leeb and Pötscher (2008a). 
some $\nu \in \mathbb{R} \cup\{-\infty, \infty\}$. [Note that in case $\zeta \neq 0$ the convergence of $n^{1 / 2} \theta_{n}$ already follows from that of $\theta_{n} / \eta_{n}$, and $\nu$ is then given by $\nu=\operatorname{sign}(\zeta) \infty$.]

1. If $|\zeta|<a$, or if $|\zeta|=a$ and $n^{1 / 2}\left(a \eta_{n}-\operatorname{sign}(\zeta) \theta_{n}\right) \rightarrow \infty$, then $F_{S C A D, n, \theta_{n}}$ approaches pointmass at $-\nu$. In case $|\nu|<\infty$, this means that $F_{S C A D, n, \theta_{n}}$ converges weakly to pointmass at $-\nu$; in case $|\nu|=\infty$, it means that the total mass of $F_{S C A D, n, \theta_{n}}$ escapes to $-\nu$, in the sense that $F_{S C A D, n, \theta_{n}}(x) \rightarrow$ 0 for every $x \in \mathbb{R}$ if $-\nu=\infty$, and $F_{S C A D, n, \theta_{n}}(x) \rightarrow 1$ for every $x \in \mathbb{R}$ if $-\nu=-\infty$.

2. If $|\zeta|=a$ and $n^{1 / 2}\left(a \eta_{n}-\operatorname{sign}(\zeta) \theta_{n}\right) \rightarrow r$ for some $r \in \mathbb{R} \cup\{-\infty\}$, then $F_{S C A D, n, \theta_{n}}(x)$ converges to

$$
\begin{gathered}
\int_{-\infty}^{x} \frac{a-2}{a-1} \phi(\{(a-2) u+\operatorname{sign}(\zeta) r\} /(a-1)) \mathbf{1}(\operatorname{sign}(\zeta) u \leq r) \\
+\phi(u) \mathbf{1}(\operatorname{sign}(\zeta) u>r) d u
\end{gathered}
$$

for every $x \in \mathbb{R}$, with the convention that the integral over the first term in the above expression is zero if $r=-\infty$. [In fact, convergence is in total variation distance.]

3. If $a<|\zeta| \leq \infty$, then $F_{S C A D, n, \theta_{n}}$ converges weakly to the standard normal distribution $N(0,1)$. [In fact, convergence is in total variation distance.]

Proof. For each $\theta$, the $\operatorname{cdf} F_{S C A D, n, \theta}$ consists of contributions from the atomic part and from the absolutely continuous part. The contribution of the absolutely continuous part can be further broken down into the contributions from the integrands $f_{1}, f_{2}, f_{3}, f_{-1}, f_{-2}$, and $f_{-3}$ in view of $(7)$. We hence may write

$$
\begin{aligned}
F_{S C A D, n, \theta}(x)=F_{0, n, \theta}(x) & +F_{1, n, \theta}(x)+F_{2, n, \theta}(x)+F_{3, n, \theta}(x) \\
& +F_{-1, n, \theta}(x)+F_{-2, n, \theta}(x)+F_{-3, n, \theta}(x),
\end{aligned}
$$

where $F_{0, n, \theta}$ denotes the contribution of the atomic part, and where the remaining terms on the right-hand side denote the contributions corresponding to $f_{1}$, $f_{2}, f_{3}, f_{-1}, f_{-2}$, and $f_{-3}$, respectively; e.g., $F_{1, n, \theta}(x)=\int_{-\infty}^{x} f_{1}(u) d u$. Now $F_{1, n, \theta_{n}}(x)$ can be written as

$$
F_{1, n, \theta_{n}}(x)=\int_{-\infty}^{x+n^{1 / 2} \eta_{n}} \phi(z) \mathbf{1}\left(n^{1 / 2}\left(\eta_{n}-\theta_{n}\right)<z \leq n^{1 / 2}\left(2 \eta_{n}-\theta_{n}\right)\right) d z .
$$

[Use the formula for $f_{1}(u)$ given after (7) with $\theta_{n}$ in place of $\theta$, and perform a simple change of variables.] By a similar argument, we also have

$$
\begin{aligned}
F_{2, n, \theta_{n}}(x)= & \int_{-\infty}^{\left((a-2) x+n^{1 / 2}\left(a \eta_{n}-\theta_{n}\right)\right) /(a-1)} \phi(z) \\
& \times \mathbf{1}\left(n^{1 / 2}\left(2 \eta_{n}-\theta_{n}\right)<z \leq n^{1 / 2}\left(a \eta_{n}-\theta_{n}\right)\right) d z,
\end{aligned}
$$


and $F_{3, n, \theta_{n}}(x)=\int_{-\infty}^{x} \phi(z) \mathbf{1}\left(z>n^{1 / 2}\left(a \eta_{n}-\theta_{n}\right)\right) d z$.

Assume first that $0 \leq \zeta<a$. In the subcase $0 \leq \zeta<1$, Proposition 1 shows that the total mass of the atomic part of $F_{S C A D, n, \theta_{n}}$ converges to one, and the statement in part 1 then follows, since $n^{1 / 2} \theta_{n} \rightarrow \nu$. For the remaining subcases to be considered observe that we have $n^{1 / 2} \theta_{n} \rightarrow \nu=\infty$ whenever $\zeta>0$. For the subcase $\zeta=1$, assume for now also that $n^{1 / 2}\left(\eta_{n}-\theta_{n}\right) \rightarrow r \in \mathbb{R} \cup\{-\infty, \infty\}$. Then the atomic part of $F_{S C A D, n, \theta_{n}}$ escapes to $-\nu=-\infty$, and the total mass of the atomic part converges to $\Phi(r)$ by Proposition 1 . In other words, $F_{0, n, \theta_{n}}(x) \rightarrow$ $\Phi(r)$ for each $x \in \mathbb{R}$, where $F_{0, n, \theta_{n}}$ denotes the contribution from the atomic part of $F_{S C A D, n, \theta_{n}}$. Moreover, from the preceding formula for $F_{1, n, \theta_{n}}(x)$, it is evident that $F_{1, n, \theta_{n}}(x) \rightarrow \int_{r}^{\infty} \phi(z) d z=1-\Phi(r)$ holds for each $x \in \mathbb{R}$ (because the upper limit in the integral diverges to $\infty$, because the lower limit in the indicator is $n^{1 / 2}\left(\eta_{n}-\theta_{n}\right) \rightarrow r$, and because the upper limit is $n^{1 / 2}\left(2 \eta_{n}-\theta_{n}\right) \rightarrow$ $\infty)$. Hence, $F_{S C A D, n, \theta_{n}}(x) \geq F_{0, n, \theta_{n}}(x)+F_{1, n, \theta_{n}}(x) \rightarrow 1$ for each $x \in \mathbb{R}$, as required. Because that limit does not depend on $r$, and because any subsequence contains a further subsequence along which $n^{1 / 2}\left(\eta_{n}-\theta_{n}\right)$ converges to some limit $r \in \mathbb{R} \cup\{-\infty, \infty\}$ (due to compactness of this space), the result follows for the subcase $\zeta=1$. In the subcase $1<\zeta<2$, it is easy to see that $F_{1, n, \theta_{n}}(x)$ converges to one for each $x \in \mathbb{R}$, whence $F_{S C A D, n, \theta_{n}}(x) \geq F_{1, n, \theta_{n}}(x) \rightarrow 1$ for each $x \in \mathbb{R}$. In the subcase $\zeta=2$, assume for now also that $n^{1 / 2}\left(2 \eta_{n}-\theta_{n}\right) \rightarrow r \in$ $\mathbb{R} \cup\{-\infty, \infty\}$. We then see that $F_{1, n, \theta_{n}}(x) \rightarrow \Phi(r)$ and $F_{2, n, \theta_{n}}(x) \rightarrow 1-\Phi(r)$, whence $F_{S C A D, n, \theta_{n}}(x) \rightarrow 1$ for each $x \in \mathbb{R}$. Because this limit does not depend on $r$ and $\mathbb{R} \cup\{-\infty, \infty\}$ is compact, a subsequence argument as above shows that the statement follows also in this subcase. Finally, in the subcases $2<\zeta<a$ and $\zeta=a$ but $n^{1 / 2}\left(a \eta_{n}-\theta_{n}\right) \rightarrow \infty$, it suffices to note that $F_{2, n, \theta_{n}}(x) \rightarrow 1$ for all $x \in \mathbb{R}$.

Assume next that $\zeta=a$ and that $n^{1 / 2}\left(a \eta_{n}-\theta_{n}\right) \rightarrow r \in \mathbb{R} \cup\{-\infty\}$. Note that $n^{1 / 2}\left(2 \eta_{n}-\theta_{n}\right)=n^{1 / 2} \eta_{n}\left(2-\theta_{n} / \eta_{n}\right) \rightarrow-\infty$ holds because $\zeta=a>2$. Using the formula for $f_{2}(u)$ and $f_{3}(u)$ given after (7) with $u$ replacing $x$ and $\theta_{n}$ replacing $\theta$, it is then easy to see that $f_{2}(u)+f_{3}(u)$ converges to the integrand in the display given in part 2, for almost all $u$. Moreover, the total mass of $F_{2, n, \theta_{n}}+F_{3, n, \theta_{n}}$ is also easily computed and seen to converge to one. Furthermore, it is easily checked that the total mass of the limiting cdf displayed in part 2 is one. Scheffe's Lemma then shows that $F_{2, n, \theta_{n}}+F_{3, n, \theta_{n}}$, and hence $F_{S C A D, n, \theta_{n}}$, converge in total variation to the limit cdf given in part 2 .

Next, assume that $\zeta>a$. Then the integrand in the formula for $F_{3, n, \theta_{n}}(x)$ converges to the density $\phi(z)$ for each $z$. The Dominated Convergence Theorem then establishes the convergence of $F_{3, n, \theta_{n}}$, and hence of $F_{S C A D, n, \theta_{n}}$, to $\Phi$ in total variation distance.

For $\zeta<0$, the proof is, mutatis mutandis, the same with $f_{-1}, f_{-2}$, and $f_{-3}$ now taking the roles of $f_{1}, f_{2}$, and $f_{3}$, respectively, and with the case $-a<\zeta \leq-1$ now being handled by showing that $1-F_{S C A D, n, \theta_{n}}(x) \rightarrow 1$ for each $x \in \mathbb{R}$. Alternatively, it can be reduced to what has already been established by observing that $F_{S C A D, n, \theta_{n}}(x)=1-F_{S C A D, n,-\theta_{n}}(-x-)$, where $F_{S C A D, n,-\theta_{n}}(\cdot-)$ denotes the limit from the left of $F_{S C A D, n,-\theta_{n}}$ at the indicated argument. 
The fixed-parameter asymptotic distribution of the SCAD estimator, including the 'oracle' property discussed at the beginning of this section, can clearly be recovered from Theorem 11 by setting $\theta_{n} \equiv \theta$. Like in the case of the hard-thresholding estimator, Theorem 11 shows that the asymptotic behavior of the SCAD-estimator is much more complicated than what the 'oracle' property predicts. In particular, Theorem 11 shows that the SCAD-estimator is not uniformly $n^{1 / 2}$-consistent. [For a discussion of the behavior of this estimator under a different scaling see the next paragraph as well as the appendix.] Furthermore, since the finite-sample distribution of the SCAD-estimator is highly non-normal but the pointwise asymptotic distribution is normal, the latter cannot adequately capture many of the essential features of the former. In contrast, the asymptotic distributions given in Theorem 11 are non-normal in some cases. All this again shows that the 'oracle' property is more of an artifact of the asymptotic framework than of much statistical significance.

The observation, that the estimators $\hat{\theta}_{H}$ and $\hat{\theta}_{S C A D}$ are not uniformly $n^{1 / 2}$ consistent if tuned to perform consistent model selection, prompts the question of the behavior of $c_{n}\left(\hat{\theta}_{H}-\theta\right)$ and $c_{n}\left(\hat{\theta}_{S C A D}-\theta\right)$ under a sequence of norming constants $c_{n}$ that are $o\left(n^{1 / 2}\right)$. Since both estimators are pointwise $n^{1 / 2}$ consistent, it follows that the pointwise limiting distributions of $c_{n}\left(\hat{\theta}_{H}-\theta\right)$ and $c_{n}\left(\hat{\theta}_{S C A D}-\theta\right)$ will then degenerate to pointmass at zero. Furthermore, it is not difficult to see that under general 'moving parameter' asymptotics the finite-sample distributions of $c_{n}\left(\hat{\theta}_{H}-\theta_{n}\right)$ and $c_{n}\left(\hat{\theta}_{S C A D}-\theta_{n}\right)$ are then nevertheless stochastically unbounded for certain sequences of parameters $\theta_{n}$ unless $c_{n}=O\left(\eta_{n}^{-1}\right)$. If $c_{n}=O\left(\eta_{n}^{-1}\right)$, Theorem 2 has shown that $c_{n}\left(\hat{\theta}_{H}-\theta_{n}\right)$ and $c_{n}\left(\hat{\theta}_{S C A D}-\theta_{n}\right)$ are indeed stochastically bounded. Hence, the uniform convergence rate of $\hat{\theta}_{H}$ and $\hat{\theta}_{S C A D}$ is seen to be given precisely by $\eta_{n}$. The precise limit distributions of these estimators under a scaling by $c_{n}$ can be obtained in a manner similar to the above theorems and are given in Theorems 17 and 19 in the appendix for the (only interesting) case $c_{n}=\eta_{n}^{-1}$. It turns out that the limit distributions under 'moving parameter' asymptotics are always given by a linear combination of at most two pointmasses, each located in the interval $[-1,1]$. With regard to the soft-thresholding estimator we have already observed that it is not even pointwise $n^{1 / 2}$-consistent. Even the distributions of $c_{n}\left(\hat{\theta}_{S}-\theta\right)$ with $c_{n}=o\left(n^{1 / 2}\right)$ are stochastically unbounded if $\theta \neq 0$ unless $c_{n}=O\left(\eta_{n}^{-1}\right)$. This is most easily seen by using the relation to the hard-thresholding estimator given in 22. If $c_{n}=O\left(\eta_{n}^{-1}\right)$, relation (2) also shows that $c_{n}\left(\hat{\theta}_{S}-\theta\right)$ is stochastically bounded, but has a degenerate (pointwise) limiting distribution. This has been noted by Zou (2006). In view of Theorem 2 under this condition on $c_{n}$ the distributions of $c_{n}\left(\hat{\theta}_{S}-\theta_{n}\right)$ are in fact stochastically bounded for any sequence $\theta_{n}$. The precise forms of the possible limit distributions under such a 'moving parameter' asymptotic are given in Theorem 18 in the appendix.

Theorems 9 and 11 demonstrate that the 'oracle' property of the hardthresholding estimator and of the SCAD-estimator paints a misleading picture of the actual finite-sample behavior of these estimators due to nonuniformity problems. In order to rescue the 'oracle' property, sometimes the argument is 
put forward that parameter sequences $\theta_{n}$ that are responsible for the nonuniformity problem should be eliminated from the parameter space a priori, since such $\theta_{n}$ are supposedly close to zero and hence are difficult to distinguish statistically from zero. While we think that such a reasoning is not sensible (because asymptotic properties of statistical procedures that are quite unstable under local perturbations of the parameter are highly suspect), we next show that the suggested reasoning actually is flawed: Consider first the consistently tuned SCAD-estimator. Suppose one considers a priori the restricted parameter space $\Theta_{n}$ of the form $\Theta_{n}=\left\{\theta: \theta=0\right.$ or $\left.|\theta| \geq b_{n}\right\}$ for some sequence $b_{n}>0$. In order to achieve that for every $\theta_{n} \in \Theta_{n}$ with $\theta_{n} \neq 0$, the distribution of $n^{1 / 2}\left(\hat{\theta}_{S C A D}-\theta_{n}\right)$ converges weakly to the standard normal $N(0,1)$ (as desired when attempting to rescue the 'oracle' property), it follows from Theorem 11 that $b_{n}$ would have to satisfy $n^{1 / 2} \eta_{n}\left(a-b_{n} / \eta_{n}\right) \rightarrow-\infty$ (e.g., $b_{n} \equiv b \eta_{n}$ with $b>a)$. But then it is easy to see that the 'forbidden' set $\mathbb{R} \backslash \Theta_{n}$ contains elements $\theta_{n}$ that are large in the sense that (i) they are of order larger than $n^{-1 / 2}$ and (ii) they are classified as nonzero with probability converging to unity by the very same SCAD-procedure, i.e., $P_{n, \theta_{n}}\left(\hat{\theta}_{S C A D} \neq 0\right) \rightarrow 1$ holds (to see this use Proposition 11). On top of this, the parameter space $\Theta_{n}$ is highly artificial, depends on sample size, and also on the tuning parameter $\eta_{n}$ and thus on the estimation procedure used. An analogous statement holds for the hardthresholding estimator (with the exception that the 'forbidden' set in this case contains $\theta_{n}$ that are large in the sense that they satisfy (i) above and (ii) are classified as non-zero with probability tending to unity by any conservatively tuned hard-thresholding procedure). Taken together, this shows that adopting a parameter space like $\Theta_{n}$ rules out values of $\theta$ that are substantially large, and not only values of $\theta$ that are statistically difficult to distinguish from zero. Hence, there seems to be little support for adopting such $\Theta_{n}$ as the parameter space.

Remark 12 The theorems in this subsection actually completely describe the limiting behavior of the finite-sample distributions of $\hat{\theta}_{H}, \hat{\theta}_{S}$, and $\hat{\theta}_{S C A D}$ without any condition on the sequence of parameters $\theta_{n}$. To see this, just apply the theorems to subsequences and note that by compactness of $\mathbb{R} \cup\{-\infty, \infty\}$ we can select from each subsequence a further subsequence such that the relevant quantities like $n^{1 / 2} \theta_{n}, \theta_{n} / \eta_{n}, n^{1 / 2}\left(\eta_{n}-\theta_{n}\right), n^{1 / 2}\left(\eta_{n}+\theta_{n}\right)$, etc. converge in $\mathbb{R} \cup\{-\infty, \infty\}$ along this further subsequence.

Remark 13 (i) As a point of interest we note that the full complexity of the possible limiting distributions in Theorems 9, 10, and 11 already arises if we restrict the sequences $\theta_{n}$ to a bounded neighborhood of zero. Hence, the phenomena described by these theorems are of a local nature, and are not tied in any way to the unboundedness of the parameter space.

(ii) It is also interesting to observe that what governs the different cases, in Theorems 9 and 11 is essentially the behavior of $\theta_{n} / \eta_{n}$, which is of smaller order than $n^{1 / 2} \theta_{n}$ because $n^{1 / 2} \eta_{n} \rightarrow \infty$ in the consistent case. Hence, an analysis relying on the usual local asymptotics based on perturbations of $\theta$ of 
the order of $n^{-1 / 2}$ does not properly reveal all possible limits of the finitesample distributions in the case where the estimators perform consistent model selection.

Remark 14 Similar as in Section 5.2.1, the mathematical reason for the failure of the pointwise asymptotic distribution to capture the behavior of the finitesample distribution well is that the convergence of the latter to the former is not uniform in the underlying parameter $\theta$. See Leeb and Pötscher (2003, 2005) for more discussion in the context of post-model-selection estimators.

\section{Impossibility results for estimating the distri- bution of $\hat{\theta}_{H}, \hat{\theta}_{S}$, and $\hat{\theta}_{S C A D}$}

As shown in Section 5.1 the $\operatorname{cdfs} F_{H, n, \theta}, F_{S, n, \theta}$, and $F_{S C A D, n, \theta}$ of the (centered and scaled) estimators $\theta_{H}, \hat{\theta}_{S}$, and $\hat{\theta}_{S C A D}$ depend on the unknown parameter $\theta$ in a complicated manner. It is hence of interest to consider estimation of these cdfs. We show that this is an intrinsically difficult estimation problem in the sense that these cdfs can not be estimated in a uniformly consistent fashion. Parts of the results that follow have been presented in earlier work (in slightly different settings): For a general class of post-model-selection estimators including the hard-thresholding estimator, this phenomenon was discussed in Leeb and Pötscher $(2006 \mathrm{~b}, 2008 \mathrm{~b})$ for the case where the estimator is tuned to be conservative, whereas Leeb and Pötscher (2006a) consider the case where the hard-thresholding estimator is tuned to be consistent; the latter paper also gives similar results for a soft-thresholding estimator tuned to be conservative. In the following, we give a simple unified treatment of hard-thresholding, softthresholding, and also of the SCAD estimator. For the SCAD estimator and for the consistently tuned soft-thresholding estimator, such non-uniformity phenomena in estimating the estimator's cdf have not been established before. We provide large-sample results that cover both consistent and conservative choices of the tuning parameter, as well as finite-sample results that hold for any choice of tuning parameter.

It is straight-forward to construct consistent estimators for the distributions of the (centered and scaled) estimators $\hat{\theta}_{H}, \hat{\theta}_{S}$ and $\hat{\theta}_{S C A D}$. One popular choice is to use subsampling or the $m$ out of $n$ bootstrap with $m / n \rightarrow 0$. Another possibility is to use the pointwise large-sample limit distributions derived in Section 5.2 together with a properly chosen pre-test of the hypothesis $\theta=0$ versus $\theta \neq 0$ : Because the pointwise large-sample limit distribution takes only two different functional forms depending on whether $\theta=0$ or $\theta \neq 0$, one can perform a pre-test that rejects the hypothesis $\theta=0$ in case $|\bar{y}|>n^{-1 / 4}$, say, and estimate the finite-sample distribution by that large-sample limit formula that corresponds to the outcome of the pre-test 9 the test's critical value $n^{-1 / 4}$

\footnotetext{
${ }^{9}$ In the consevative case, the asymptotic distribution can also depend on $e$ which is then to be replaced by $n^{1 / 2} \eta_{n}$.
} 
ensures that the correct large-sample limit formula is selected with probability approaching one as sample size increases.

When estimating the distribution of thresholding (and related) estimators, there is evidence in the literature that certain specific consistent estimation procedures, like those sketched above, may not perform well in a worst-case scenario. For some examples, see Kulperger and Ahmed (1992); the disclaimer issued after Corollary 2.1 in Beran (1997); the discussion at the end of Section 4 in Knight and Fu (2000); or Samworth (2003). The next result shows that this problem is not caused by the specifics of the consistent estimators under consideration but is an intrinsic feature of the estimation problem itself.

Theorem 15 Let $\hat{\theta}$ denote any one of the estimators $\hat{\theta}_{H}, \hat{\theta}_{S}$, or $\hat{\theta}_{S C A D}$, and write $F_{n, \theta}$ for the cdf of $n^{1 / 2}(\hat{\theta}-\theta)$ under $P_{n, \theta}$. Consider a sequence of tuning parameters such that $\eta_{n} \rightarrow 0$ and $n^{1 / 2} \eta_{n} \rightarrow e$ as $n \rightarrow \infty$ with $0 \leq e \leq \infty$. Let $t \in \mathbb{R}$ be arbitrary. Then every consistent estimator $\hat{F}_{n}(t)$ of $F_{n, \theta}(t)$ satisfies

$$
\lim _{n \rightarrow \infty} \sup _{|\theta|<c / n^{1 / 2}} P_{n, \theta}\left(\left|\hat{F}_{n}(t)-F_{n, \theta}(t)\right|>\varepsilon\right)=1
$$

for each $\varepsilon<(\Phi(t+e)-\Phi(t-e)) / 2$ and each $c>|t|$. In particular, no uniformly consistent estimator for $F_{n, \theta}(t)$ exists.

Proof. For two sequences $\theta_{n}^{(1)}$ and $\theta_{n}^{(2)}$ satisfying $\left|\theta_{n}^{(i)}\right|<c / n^{1 / 2}, i=1,2$, the probability measures $P_{n, \theta_{n}^{(1)}}$ and $P_{n, \theta_{n}^{(2)}}$ are mutually contiguous as is elementary to verify (cf., e.g., Lemma A.1 of Leeb and Pötscher (2006a)). The corresponding estimands $F_{n, \theta_{n}^{(1)}}(t)$ and $F_{n, \theta_{n}^{(2)}}(t)$, however, do not necessarily get close to each other: For each $\delta$ write $\theta_{n}(\delta)$ as shorthand for $\theta_{n}(\delta)=-(t+\delta) / n^{1 / 2}$. The cdfs $F_{n, \theta_{n}(\delta)}(\cdot)$ and $F_{n, \theta_{n}(-\delta)}(\cdot)$ have a jump at $t+\delta$ and at $t-\delta$, respectively, so that for $\delta>0$

$$
F_{n, \theta_{n}(-\delta)}(t)-F_{n, \theta_{n}(\delta)}(t)=\Phi\left(t-\delta+n^{1 / 2} \eta_{n}\right)-\Phi\left(t-\delta-n^{1 / 2} \eta_{n}\right)+r(\delta) ;
$$

cf. (4), (5), and (7) for $\hat{\theta}_{H}, \hat{\theta}_{S}$, and $\hat{\theta}_{S C A D}$, respectively. Moreover, $r(\delta)$ goes to zero with $\delta \rightarrow 0$, because the absolutely continuous part of $F_{n, \theta}(t)$ is a continuous function of $\theta$ (again in view of the finite-sample formulae and dominated convergence). Taking the supremum of $\left|F_{n, \theta_{n}(-\delta)}(t)-F_{n, \theta_{n}(\delta)}(t)\right|$ over all $\delta$ with $0 \leq \delta<c-|t|$, we obtain that this supremum is bounded from below by $\Phi\left(t+n^{1 / 2} \eta_{n}\right)-\Phi\left(t-n^{1 / 2} \eta_{n}\right)$. [To see this note that this supremum is not less than $\lim _{i \rightarrow \infty}\left|F_{n, \theta_{n}(-1 / i)}(t)-F_{n, \theta_{n}(1 / i)}(t)\right|$ and use $[11)$.] Because that lower bound converges to $\Phi(t+e)-\Phi(t-e)$ as $n \rightarrow \infty$, the theorem now follows from Lemma 3.1 of Leeb and Pötscher (2006a). [Use this result with the identifications $\beta=\theta, \varphi_{n}(\beta)=F_{n, \theta}(t), B_{n}=\left\{\theta:|\theta|<c / n^{1 / 2}\right\}, \alpha=0$, and with $d(a, b)=|a-b|$. Moreover, note that $B_{n}$ contains $\theta_{n}(\delta)$ and $\theta_{n}(-\delta)$ for $0 \leq \delta<c-|t|$.

We stress that the above result also applies to any kind of bootstrap- or subsampling-based estimator of the $\operatorname{cdf} F_{n, \theta}$ whatsoever, since the results in 
Leeb and Pötscher (2006a) on which the proof of Theorem 15 rests apply to arbitrary randomized estimators (cf. Lemma 3.6 in Leeb and Pötscher (2006a)); the same applies to Theorem 16 that follows as well as to Theorem 20 in the appendix.

Loosely speaking, Theorem 15 states that any consistent estimator for the cdf of interest suffers from an unavoidable worst-case error of at least $\varepsilon$ with $\varepsilon<(\Phi(t+e)-\Phi(t-e)) / 2$. The error range, i.e., $(\Phi(t+e)-\Phi(t-e)) / 2$, is governed by the limit $e=\lim _{n} n^{1 / 2} \eta_{n}$. In case the estimator $\hat{\theta}$ is tuned to be consistent, i.e., in case $e=\infty$, the error range equals $1 / 2$, and the phenomenon is most pronounced. If the estimator $\hat{\theta}$ is tuned to be conservative so that $e<\infty$, the error range is less than $1 / 2$ but can still be substantial. Only in case $e=0$ the error range equals zero, and the condition $\varepsilon<(\Phi(t+e)-\Phi(t-e)) / 2$ in Theorem 15 leads to a trivial conclusion. This is, however, not surprising as then the resulting estimator is uniformly asymptotically equivalent to the unrestricted maximum likelihood estimator $\bar{y}$; cf. Remark 3 .

A similar non-uniformity phenomenon as described in Theorem 15 for consistent estimators $\hat{F}_{n}(t)$ also occurs for not necessarily consistent estimators. For such arbitrary estimators, we find in the following that the phenomenon can be somewhat less pronounced, in the sense that the lower bound is now $1 / 2$ instead of 1; cf. 13 below. The following theorem gives a large-sample limit result that parallels Theorem 15, as well as a finite-sample result, both for arbitrary (and not necessarily consistent) estimators of the cdf.

Theorem 16 Let $\hat{\theta}$ denote any one of the estimators $\hat{\theta}_{H}, \hat{\theta}_{S}$, or $\hat{\theta}_{S C A D}$, and write $F_{n, \theta}$ for the cdf of $n^{1 / 2}(\hat{\theta}-\theta)$ under $P_{n, \theta}$. Let $0<\eta_{n}<\infty$ and let $t \in \mathbb{R}$ be arbitrary. Then every estimator $\hat{F}_{n}(t)$ of $F_{n, \theta}(t)$ satisfies

$$
\sup _{|\theta|<c / n^{1 / 2}} P_{n, \theta}\left(\left|\hat{F}_{n}(t)-F_{n, \theta}(t)\right|>\varepsilon\right) \geq \frac{1}{2}
$$

for each $\varepsilon<\left(\Phi\left(t+n^{1 / 2} \eta_{n}\right)-\Phi\left(t-n^{1 / 2} \eta_{n}\right)\right) / 2$, for each $c>|t|$, and for each fixed sample size $n$. If $\eta_{n}$ satisfies $\eta_{n} \rightarrow 0$ and $n^{1 / 2} \eta_{n} \rightarrow e$ as $n \rightarrow \infty$ with $0 \leq e \leq \infty$, we thus have

$$
\liminf _{n \rightarrow \infty} \inf _{\hat{F}_{n}(t)} \sup _{|\theta|<c / n^{1 / 2}} P_{n, \theta}\left(\left|\hat{F}_{n}(t)-F_{n, \theta}(t)\right|>\varepsilon\right) \geq \frac{1}{2}
$$

for each $\varepsilon<(\Phi(t+e)-\Phi(t-e)) / 2$ and for each $c>|t|$, where the infimum in 13) extends over all estimators $\hat{F}_{n}(t)$.

Proof. Only the finite-sample statement needs to be proven. Let $\theta_{n}(\delta)$ be as in the proof of Theorem 15. The total variation distance of $P_{n, \theta_{n}(\delta)}$ and $P_{n, \theta_{n}(-\delta)}$, i.e., $\left\|P_{n, \theta_{n}(\delta)}-P_{n, \theta_{n}(-\delta)}\right\|_{T V}$, goes to zero as $\delta \rightarrow 0$ (which is easy to see, either by direct computation or using, say, Lemma A.1 of Leeb and Pötscher (2006a)). In view of (11), however, the estimands $F_{n, \theta_{n}(\delta)}(t)$ and $F_{n, \theta_{n}(-\delta)}(t)$ do not get close to each other as $\delta \rightarrow 0(\delta>0)$, as we have already seen in the proof of 
Theorem 15. For each $\varepsilon$ that is smaller than $\left|F_{n, \theta_{n}(-\delta)}(t)-F_{n, \theta_{n}(\delta)}(t)\right| / 2$, the left-hand side of $\sqrt{12}$ is bounded from below by

$$
\frac{1}{2}\left(1-\left\|P_{n, \theta_{n}(\delta)}-P_{n, \theta_{n}(-\delta)}\right\|_{T V}\right) .
$$

This follows from Lemma 3.2 of Leeb and Pötscher (2006a) together with Remark B.2 of that paper. [Use the result described in Remark B.2 with $A=\{n\}$, $\beta=\theta, B_{n}=\left\{\theta_{n}(\delta), \theta_{n}(-\delta)\right\}, \varphi_{n}(\beta)=F_{n, \theta}(t), d(a, b)=|a-b|$, and with $\delta^{*}$ equal to $\left|F_{n, \theta_{n}(-\delta)}(t)-F_{n, \theta_{n}(\delta)}(t)\right|$. Moreover, note that $B_{n}$ is contained in $\left\{\theta:|\theta|<c / n^{1 / 2}\right\}$ provided $\left.0<\delta<c-|t|.\right]$ For $\delta \rightarrow 0$, now observe that the expression in the preceding display converges to $1 / 2$, i.e., the lower bound in $(12)$, and that $\left|F_{n, \theta_{n}(-\delta)}(t)-F_{n, \theta_{n}(\delta)}(t)\right|$ converges to $\Phi\left(t+n^{1 / 2} \eta_{n}\right)-\Phi\left(t-n^{1 / 2} \eta_{n}\right)$.

Apart from being of interest in its own right, the asymptotic statement in Theorem 16 also provides additional insight into some phenomena related to inference based on shrinkage-type estimators that have recently attracted some attention: When estimating the cdf of a hard-thresholding estimator, Samworth (2003) noted that, while the bootstrap is not consistent, it nevertheless may perform better, in a uniform sense, than the $m$ out of $n$ bootstrap which is consistent (provided $m \rightarrow \infty, m / n \rightarrow 0$ ). Theorem 15 and the asymptotic statement in Theorem 16 together show that this phenomenon of better performance of the bootstrap is possible precisely because the bootstrap is not consistent.

The finite-sample statement in Theorem 16 clearly reveals how the estimability of the cdf of the estimator depends on the tuning parameter $\eta_{n}$ : A larger value of $\eta_{n}$, which results in a 'more sparse' estimator in view of (1), directly corresponds to a larger range $\left(\Phi\left(t+n^{1 / 2} \eta_{n}\right)-\Phi\left(t-n^{1 / 2} \eta_{n}\right)\right) / 2$ for the error $\varepsilon$ within which any estimator $\hat{F}_{n}(t)$ performs poorly in the sense of 12$)$. In large samples, the limit $e=\lim _{n} n^{1 / 2} \eta_{n}$ takes the role of $n^{1 / 2} \eta_{n}$.

An impossibility result paralleling Theorem 16 for the cdf of $\eta_{n}^{-1}(\hat{\theta}-\theta)$, where $\hat{\theta}=\hat{\theta}_{H}, \hat{\theta}_{S}$, or $\hat{\theta}_{S C A D}$, is given in the appendix.

\section{Conclusion}

We have studied the distribution of the LASSO, i.e., of a soft-thresholding estimator, of the SCAD, and of a hard-thresholding estimator in finite samples and in the large-sample limit. The finite-sample distributions of these estimators were found to be highly non-normal, because they are a mixture of a singular normal distribution and an absolutely continuous component that can be multimodal, for example. The large-sample behavior of these distributions depends on the choice of the estimators' tuning parameter where, in essence, two cases can occur:

In the first case, the estimator can be viewed as performing conservative model selection. In this case, fixed-parameter asymptotics, where the true parameters are held fixed while sample size increases, reflect the large-sample behavior only in part. 'Moving parameter' asymptotics, where the true parameter 
may depend on sample size, give a more complete picture. We have seen that the distribution of the LASSO, of the SCAD, and of the hard-thresholding estimator can be highly non-normal irrespective of sample size, in particular in the statistically interesting case where the true parameter is close (in an appropriate sense) to a lower-dimensional submodel. This also shows that the finite-sample phenomena that we have observed are not small-sample effects but can occur at any sample size.

In the second case, the estimator can be viewed as performing consistent model selection, and the hard-thresholding as well as the SCAD estimator have the 'oracle' property in the sense of Fan and Li (2001). [This is not so for the LASSO.] This 'oracle' property, which is based on fixed-parameter asymptotics, seems to suggest that the estimator in question performs very well in large samples. However, as before, fixed-parameter asymptotics do not capture the whole range of large-sample phenomena that can occur. With 'moving parameter' asymptotics, we have shown that the distribution of these estimators can again be highly non-normal, even in large samples. In addition, we have found that the observed finite-sample phenomena not only can persist but actually can be more pronounced for larger sample sizes. For example, the distribution of the SCAD estimator can diverge in the sense that all its mass escapes to either $+\infty$ or $-\infty$.

We have also demonstrated that the LASSO, the SCAD, and the hardthresholding estimator are always uniformly consistent, irrespective of the choice of tuning parameter (except for non-sensible choices). In case the tuning is such that the estimator acts as a conservative model selector, we have also seen that these estimators are in fact uniformly $n^{1 / 2}$-consistent. However, uniform $n^{1 / 2}$ consistency no longer holds in the case where the estimator acts like a consistent model selector (and where the SCAD and the hard-thresholding estimator have the 'oracle' property). In fact, the estimators then have a uniform convergence rate slower than $n^{-1 / 2}$ in that they are only uniformly $\eta_{n}^{-1}$-consistent. The asymptotic distributions of the estimators under an $\eta_{n}^{-1}$-scaling, rather than an $n^{1 / 2}$-scaling, are discussed in the appendix.

Finally, we have studied the problem of estimating the cdf of the (centered and scaled) LASSO, SCAD, and hard-thresholding estimator. We have shown that this cdf can not be estimated in a uniformly consistent fashion, even though pointwise consistent estimators can be constructed with relative ease. Moreover, we have obtained performance bounds for estimators of the cdf that suggest that inconsistent estimators for this cdf may actually perform better, in a uniform sense, than consistent estimators.

The phenomena observed here for distributional properties of the estimators under consideration not surprisingly spill over to the estimators' risk behavior. The finite-sample distributions derived in this paper in fact facilitate a detailed risk analysis, but this is not our main focus here. Therefore, we only point out the most important risk phenomena: We consider squared error loss scaled by sample size (i.e., $\left.L(\hat{\theta}, \theta)=n(\hat{\theta}-\theta)^{2}\right)$, and we shall compare the estimators to the maximum-likelihood estimator based on the overall model, i.e., $\hat{\theta}_{U}=\bar{y}$. In finite 
samples, the LASSO, the SCAD, and the hard-thresholding estimator compare favorably with $\hat{\theta}_{U}$ in terms of risk, if the true parameter is in a neighborhood of the lower dimensional model; outside of that neighborhood, the situation is reversed. [This is well-known for the hard- and soft-thresholding estimators and for more general pre-test estimators; cf. Judge and Bock (1978), Bruce and Gao (1996). Explicit formulae for the risk of a hard-thresholding estimator are also given in Leeb and Pötscher (2005).] As sample size goes to infinity, again two cases need to be distinguished: If these estimators are tuned to perform conservative model selection, the worst-case risk of the LASSO, of the SCAD, and of the hard-thresholding estimator remains bounded as sample size increases. If the tuning is such that these estimators perform consistent model selection (the case when the SCAD as well as the hard-thresholding estimator have the 'oracle' property), then the worst-case risk of these estimators increases indefinitely as sample size goes to infinity. [In fact, this is true for any estimator that has a 'sparsity' property; see Theorem 2.1 in Leeb and Pötscher (2008a) for details.] Thus for these estimators the asymptotic worst-case risk behavior is in marked contrast to their favorable pointwise asymptotic risk behavior reflected in the 'oracle' property. For the SCAD, the LASSO, and for the hard-thresholding estimator, this worst-case risk behavior is also in line with the fact that these estimators are uniformly $n^{1 / 2}$-consistent if tuned to perform conservative model selection, but that uniform $n^{1 / 2}$-consistency breaks down when they are tuned to perform consistent model selection.

Finally we want to stress that our results should not be read as a criticism of penalized maximum likelihood estimators per se, but rather as a warning that the distributional properties of such estimators are more intricate and complex than might appear at first glance.

\section{Acknowledgments}

Input and suggestions from Ulrike Schneider are greatly appreciated.

\section{A Appendix}

For the case where the estimators $\hat{\theta}_{H}, \hat{\theta}_{S}$, and $\hat{\theta}_{S C A D}$ are tuned to perform consistent model selection (i.e., $\eta_{n} \rightarrow 0$ and $n^{1 / 2} \eta_{n} \rightarrow \infty$ ), we now consider the possible limits of the distributions of $c_{n}\left(\hat{\theta}_{H}-\theta_{n}\right), c_{n}\left(\hat{\theta}_{S}-\theta_{n}\right)$, and $c_{n}\left(\hat{\theta}_{S C A D}-\right.$ $\theta_{n}$ ) when $c_{n}=O\left(\eta_{n}^{-1}\right)$. The only interesting case is where $c_{n} \sim \eta_{n}^{-1}$, since for $c_{n}=o\left(\eta_{n}^{-1}\right)$ these limits are always pointmass at zero in view of Theorem $2{ }^{10}$ Let $G_{H, n, \theta}, G_{S, n, \theta}$, and $G_{S C A D, n, \theta}$ stand for the finite-sample distributions of $\eta_{n}^{-1}\left(\hat{\theta}_{H}-\theta\right), \eta_{n}^{-1}\left(\hat{\theta}_{S}-\theta\right)$, and $\eta_{n}^{-1}\left(\hat{\theta}_{S C A D}-\theta\right)$, respectively, under $P_{n, \theta}$. Clearly, $G_{H, n, \theta}(x)=F_{H, n, \theta}\left(n^{1 / 2} \eta_{n} x\right)$ and similar relations hold for $G_{S, n, \theta}$ and

\footnotetext{
${ }^{10}$ There is no loss in generality here in the sense that the general case where $c_{n}=O\left(\eta_{n}^{-1}\right)$ holds can - by passing to subsequences - always be reduced to the cases where $c_{n} \sim \eta_{n}^{-1}$ or $c_{n}=o\left(\eta_{n}^{-1}\right)$ holds.
} 
$G_{S C A D, n, \theta}$. We next provide the limits of these distributions under 'moving parameter' asymptotics. Note that comments like in Remarks 12, 13, and 14 also apply to the three subsequent theorems.

Theorem 17 Consider the hard-thresholding estimator with $\eta_{n} \rightarrow 0$ and $n^{1 / 2} \eta_{n} \rightarrow \infty$. Assume that $\theta_{n} / \eta_{n} \rightarrow \zeta$ for some $\zeta \in \mathbb{R} \cup\{-\infty, \infty\}$.

1. If $|\zeta|<1$, then $G_{H, n, \theta_{n}}$ converges weakly to pointmass $\delta_{-\zeta}$.

2. If $|\zeta|=1$ and $n^{1 / 2}\left(\eta_{n}-\zeta \theta_{n}\right) \rightarrow r$ for some $r \in \mathbb{R} \cup\{-\infty, \infty\}$, then $G_{H, n, \theta_{n}}$ converges weakly to

$$
\Phi(r) \delta_{-\zeta}+(1-\Phi(r)) \delta_{0}
$$

3. If $1<|\zeta| \leq \infty$, then $G_{H, n, \theta_{n}}$ converges weakly to pointmass $\delta_{0}$.

Proof. Consider case 1 first. On the event $\left\{\hat{\theta}_{H}=0\right\}$ we have $\eta_{n}^{-1}\left(\hat{\theta}_{H}-\right.$ $\left.\theta_{n}\right)=-\eta_{n}^{-1} \theta_{n}$. By Proposition 1. $P_{n, \theta_{n}}\left(\hat{\theta}_{H}=0\right) \rightarrow 1$. Since $\eta_{n}^{-1} \theta_{n} \rightarrow \zeta$ by assumption, the result follows. To prove case 2 write $\eta_{n}^{-1}\left(\hat{\theta}_{H}-\theta_{n}\right)$ as $-\eta_{n}^{-1} \theta_{n} \mathbf{1}\left(\hat{\theta}_{H}=0\right)+\left(n^{1 / 2} \eta_{n}\right)^{-1} Z_{n} \mathbf{1}\left(\hat{\theta}_{H} \neq 0\right)$ where $Z_{n}$ is standard normally distributed under $P_{n, \theta_{n}}$. Since Proposition 1 shows that $P_{n, \theta_{n}}\left(\hat{\theta}_{H}=0\right) \rightarrow \Phi(r)$, the result in case 2 now follows as is easily seen. To prove case 3 , observe that $\eta_{n}^{-1}\left(\hat{\theta}_{H}-\theta_{n}\right)=\left(n^{1 / 2} \eta_{n}\right)^{-1} n^{1 / 2}\left(\hat{\theta}_{H}-\theta_{n}\right)$ and that $n^{1 / 2}\left(\hat{\theta}_{H}-\theta_{n}\right)$ converges to a standard normal distribution under $P_{n, \theta_{n}}$ in view of Theorem 9

Theorem 18 Consider the soft-thresholding estimator with $\eta_{n} \rightarrow 0$ and $n^{1 / 2} \eta_{n} \rightarrow \infty$. Assume that $\theta_{n} / \eta_{n} \rightarrow \zeta$ for some $\zeta \in \mathbb{R} \cup\{-\infty, \infty\}$. Then $G_{S, n, \theta_{n}}$ converges weakly to pointmass $\delta_{-\operatorname{sign}(\zeta) \min (1,|\zeta|)}$.

Proof. From (6) we obtain that

$G_{S, n, \theta_{n}}(x)=\Phi\left(n^{1 / 2} \eta_{n}(x+1)\right) \mathbf{1}\left(x \geq-\theta_{n} / \eta_{n}\right)+\Phi\left(n^{1 / 2} \eta_{n}(x-1)\right) \mathbf{1}\left(x<-\theta_{n} / \eta_{n}\right)$.

Now it is easy to see that this expression converges to 0 if $x<$ $-\operatorname{sign}(\zeta) \min (1,|\zeta|)$ and to 1 if $x>-\operatorname{sign}(\zeta) \min (1,|\zeta|)$.

Theorem 19 Consider the SCAD estimator with $\eta_{n} \rightarrow 0$ and $n^{1 / 2} \eta_{n} \rightarrow \infty$. Assume that $\theta_{n} / \eta_{n} \rightarrow \zeta$ for some $\zeta \in \mathbb{R} \cup\{-\infty, \infty\}$.

1. If $|\zeta| \leq 2$, then $G_{S C A D, n, \theta_{n}}$ converges weakly to pointmass $\delta_{-\operatorname{sign}(\zeta) \min (1,|\zeta|)}$.

2. If $2<|\zeta|<a$, then $G_{S C A D, n, \theta_{n}}$ converges weakly to pointmass $\delta_{-\operatorname{sign}(\zeta)(a-|\zeta|) /(a-2)}$.

3. If $a \leq|\zeta| \leq \infty$, then $G_{S C A D, n, \theta_{n}}$ converges weakly to pointmass $\delta_{0}$. 
Proof. If $|\zeta|<1$ the proof is identical to the proof of case 1 in Theorem 17. Next assume $\zeta=1$ : assume also for the moment that $n^{1 / 2}\left(\eta_{n}-\theta_{n}\right) \rightarrow r$, $r \in \mathbb{R} \cup\{-\infty, \infty\}$. The atomic part $G_{0, n, \theta_{n}}$ of the $\operatorname{cdf} G_{S C A D, n, \theta_{n}}(x)$ is given by $\left\{\Phi\left(n^{1 / 2}\left(-\theta_{n}+\eta_{n}\right)\right)-\Phi\left(n^{1 / 2}\left(-\theta_{n}-\eta_{n}\right)\right)\right\} \mathbf{1}\left(x \geq-\theta_{n} / \eta_{n}\right)$ which is seen to converge weakly to $\Phi(r) \mathbf{1}(x \geq-1)$ which is the cdf of $\Phi(r) \delta_{-1}$. Furthermore, recalling the definition of $F_{i, n, \theta}$ given in the proof of Theorem 11 .

$$
\begin{aligned}
G_{1, n, \theta_{n}}(x) & =F_{1, n, \theta_{n}}\left(n^{1 / 2} \eta_{n} x\right) \\
& =\int_{-\infty}^{n^{1 / 2} \eta_{n}(x+1)} \phi(z) \mathbf{1}\left(n^{1 / 2}\left(\eta_{n}-\theta_{n}\right)<z \leq n^{1 / 2}\left(2 \eta_{n}-\theta_{n}\right)\right) d z
\end{aligned}
$$

is seen to converge to 0 for $x<-1$ and to $1-\Phi(r)$ for $x>-1$, since $n^{1 / 2}\left(\eta_{n}-\theta_{n}\right) \rightarrow r$ and $n^{1 / 2}\left(2 \eta_{n}-\theta_{n}\right)=n^{1 / 2} \eta_{n}\left(2-\theta_{n} / \eta_{n}\right) \rightarrow \infty$. Hence, $G_{1, n, \theta_{n}}$ converges weakly to $(1-\Phi(r)) \delta_{-1}$, and thus $G_{0, n, \theta_{n}}+G_{1, n, \theta_{n}}$ converges weakly to pointmass $\delta_{-1}$. This implies that also $G_{S C A D, n, \theta_{n}}$ has the same limit. Since the limit does not depend on $r$, a subsequence argument as in the proof of Theorem 11 completes the proof of the case $\zeta=1$. Next consider the case $1<\zeta<2$ : Here $G_{1, n, \theta_{n}}(x)$ is easily seen to converge to 0 for $x<-1$ and to 1 for $x>-1$, since $n^{1 / 2}\left(\eta_{n}-\theta_{n}\right)=n^{1 / 2} \eta_{n}\left(1-\theta_{n} / \eta_{n}\right) \rightarrow-\infty$ and $n^{1 / 2}\left(2 \eta_{n}-\theta_{n}\right)=n^{1 / 2} \eta_{n}\left(2-\theta_{n} / \eta_{n}\right) \rightarrow \infty$. Hence, $G_{1, n, \theta_{n}}$ converges weakly to pointmass $\delta_{-1}$, and consequently $G_{S C A D, n, \theta_{n}}$ has to have the same limit. We turn to the case $\zeta=2$ : Assume now for the moment that $n^{1 / 2}\left(2 \eta_{n}-\theta_{n}\right) \rightarrow r$, $r \in \mathbb{R} \cup\{-\infty, \infty\}$. Then $G_{1, n, \theta_{n}}(x)$ is seen to converge to 0 for $x<-1$ and to $\Phi(r)$ for $x>-1$, since $n^{1 / 2}\left(\eta_{n}-\theta_{n}\right)=n^{1 / 2} \eta_{n}\left(1-\theta_{n} / \eta_{n}\right) \rightarrow-\infty$ and $n^{1 / 2}\left(2 \eta_{n}-\theta_{n}\right) \rightarrow r$. Furthermore, note that in the case considered $\left((a-2) n^{1 / 2} \eta_{n} x+n^{1 / 2}\left(a \eta_{n}-\theta_{n}\right)\right) /(a-1)$ converges to $-\infty$ for $x<-1$ and to $\infty$ for $x>-1$. Consequently,

$$
\begin{aligned}
G_{2, n, \theta_{n}}(x)= & F_{2, n, \theta_{n}}\left(n^{1 / 2} \eta_{n} x\right) \\
= & \int_{-\infty}^{\left((a-2) n^{1 / 2} \eta_{n} x+n^{1 / 2}\left(a \eta_{n}-\theta_{n}\right)\right) /(a-1)} \phi(z) \\
& \quad \times \mathbf{1}\left(n^{1 / 2}\left(2 \eta_{n}-\theta_{n}\right)<z \leq n^{1 / 2}\left(a \eta_{n}-\theta_{n}\right)\right) d z
\end{aligned}
$$

is seen to converge to 0 for $x<-1$ and to $1-\Phi(r)$ for $x>-1$, since $n^{1 / 2}\left(2 \eta_{n}-\theta_{n}\right) \rightarrow r$ and $n^{1 / 2}\left(a \eta_{n}-\theta_{n}\right)=n^{1 / 2} \eta_{n}\left(a-\theta_{n} / \eta_{n}\right) \rightarrow \infty$. But this shows that $G_{1, n, \theta_{n}}+G_{2, n, \theta_{n}}$ converges weakly to pointmass $\delta_{-1}$, and hence the same must be true for $G_{S C A D, n, \theta_{n}}$. Since the limit does not depend on $r$, a subsequence argument completes the proof for the case $\zeta=2$. Consider next the case where $2<\zeta<a$ : Then $G_{2, n, \theta_{n}}(x)$ is easily seen to converge to 0 if $x<-(a-\zeta) /(a-2)$ and to 1 if $x>-(a-\zeta) /(a-2)$, since $\left((a-2) n^{1 / 2} \eta_{n} x+n^{1 / 2}\left(a \eta_{n}-\theta_{n}\right)\right) /(a-1)$ converges to $-\infty$ or $\infty$ depending on whether $x$ is smaller or larger than $-(a-\zeta) /(a-2)$, and since $n^{1 / 2}\left(2 \eta_{n}-\theta_{n}\right) \rightarrow$ $-\infty$ and $n^{1 / 2}\left(a \eta_{n}-\theta_{n}\right) \rightarrow \infty$. This proves that $G_{2, n, \theta_{n}}$, and hence $G_{S C A D, n, \theta_{n}}$, converges weakly to pointmass $\delta_{-(a-\zeta) /(a-2)}$. Assume next that $\zeta=a$ and assume for the moment that $n^{1 / 2}\left(a \eta_{n}-\theta_{n}\right) \rightarrow r, r \in \mathbb{R} \cup\{-\infty, \infty\}$ : Then the 
upper limit in the integral defining $G_{2, n, \theta_{n}}$ converges to $\infty$ if $x>0$ and to $-\infty$ if $x<0$. This is obvious if $|r|<\infty$, and follows from rewriting the upper limit as $n^{1 / 2} \eta_{n}\left((a-2) x+a-\theta_{n} / \eta_{n}\right) /(a-1)$ if $|r|=\infty$. Furthermore, the lower limit in the indicator function in 14 converges to $-\infty$, while the upper limit converges to $r$. This shows that $G_{2, n, \theta_{n}}$ converges weakly to $\Phi(r) \delta_{0}$. Inspection of $G_{3, n, \theta_{n}}(x)=F_{3, n, \theta_{n}}\left(n^{1 / 2} \eta_{n} x\right)=\int_{-\infty}^{n^{1 / 2} \eta_{n} x} \phi(z) \mathbf{1}\left(z>n^{1 / 2}\left(a \eta_{n}-\theta_{n}\right)\right) d z$ shows that this converges weakly to $(1-\Phi(r)) \delta_{0}$. Together this gives weak convergence of $G_{2, n, \theta_{n}}+G_{3, n, \theta_{n}}$, and hence of $G_{S C A D, n, \theta_{n}}$, to pointmass $\delta_{0}$. Since the limit does not depend on $r$, a subsequence argument again completes the proof of the case $\zeta=a$. Suppose next that $a<\zeta \leq \infty$ : Inspection of $G_{3, n, \theta_{n}}$ immediately shows that it (and hence also $G_{S C A D, n, \theta_{n}}$ ) converges weakly to pointmass $\delta_{0}$. The remaining cases for $\zeta \leq-1$ are proved completely analogous to the corresponding cases with positive $\zeta$.

Finally, we provide an impossibility result for the estimation of the finite sample distributions $G_{H, n, \theta}, G_{S, n, \theta}$, and $G_{S C A D, n, \theta}$.

Theorem 20 Let $\hat{\theta}$ denote any one of the estimators $\hat{\theta}_{H}, \hat{\theta}_{S}$, or $\hat{\theta}_{S C A D}$, and write $G_{n, \theta}$ for the cdf of $\eta_{n}^{-1}(\hat{\theta}-\theta)$ under $P_{n, \theta}$. Let $0<\eta_{n}<\infty$ and let $t \in \mathbb{R}$ be arbitrary. Then every estimator $\hat{G}_{n}(t)$ of $G_{n, \theta}(t)$ satisfies

$$
\sup _{|\theta|<c \eta_{n}} P_{n, \theta}\left(\left|\hat{G}_{n}(t)-G_{n, \theta}(t)\right|>\varepsilon\right) \geq \frac{1}{2}
$$

for each $\varepsilon<\left(\Phi\left(n^{1 / 2} \eta_{n}(t+1)\right)-\Phi\left(n^{1 / 2} \eta_{n}(t-1)\right)\right) / 2$, for each $c>|t|$, and for each fixed sample size $n$. If $\eta_{n}$ satisfies $\eta_{n} \rightarrow 0$ and $n^{1 / 2} \eta_{n} \rightarrow \infty$ as $n \rightarrow \infty$, we thus have for each $c>|t|$

$$
\liminf _{n \rightarrow \infty} \inf _{\hat{G}_{n}(t)|\theta|<c \eta_{n}} \sup _{n, \theta}\left(\left|\hat{G}_{n}(t)-G_{n, \theta}(t)\right|>\varepsilon\right) \geq \frac{1}{2}
$$

for each $\varepsilon<1 / 2$ if $|t|<1$ and for $\varepsilon<1 / 4$ if $|t|=1$, where the infimum in (16) extends over all estimators $\hat{G}_{n}(t)$.

This result shows, in particular, that no uniformly consistent estimator exists for $G_{n, \theta}(t)$ in case $|t| \leq 1$ (not even over compact subsets of $\mathbb{R}$ containing the origin). In view of Theorems 17, 18, and 19 we see that for $t>1$ we have $\sup _{\theta \in \mathbb{R}}\left|G_{n, \theta}(t)-1\right| \rightarrow 0$ as $n \rightarrow \infty$, hence $G_{n}(t)=1$ is trivially a uniformly consistent estimator. Similarly, for $t<-1$ we have $\sup _{\theta \in \mathbb{R}}\left|G_{n, \theta}(t)\right| \rightarrow 0$ as $n \rightarrow \infty$, hence $\hat{G}_{n}(t)=0$ is trivially a uniformly consistent estimator.

Proof. We first prove 15 . For fixed $n$ and $t$ set $s=n^{1 / 2} \eta_{p} t$. Define $\hat{F}_{n}(s)=$ $\hat{G}_{n}(t)$. Also note that $G_{n, \theta}(t)=F_{n, \theta}(s)$ holds. By Theorem 16 we know that

$$
\sup _{|\theta|<d / n^{1 / 2}} P_{n, \theta}\left(\left|\hat{F}_{n}(s)-F_{n, \theta}(s)\right|>\varepsilon\right) \geq \frac{1}{2}
$$

for each $\varepsilon<\left(\Phi\left(s+n^{1 / 2} \eta_{n}\right)-\Phi\left(s-n^{1 / 2} \eta_{n}\right)\right) / 2$ and for each $d>|s|$. Rewriting this in terms of $t, \hat{G}_{n}(t)$, and $G_{n, \theta}(t)$ and setting $c=d n^{-1 / 2} / \eta_{n}$ gives (15). Relation 16 is a trivial consequence of 15 . 


\section{References}

Bauer, P., Pötscher, B. M. \& P. Hackl (1988): Model selection by multiple test procedures. Statistics 19, 39-44.

Beran, R. (1997): Diagnosing bootstrap success. Annals of the Institute of Statistical Mathematics 49, 1-24.

Bruce, A. G. \& H. Gao (1996): Understanding WaveShrink: Variance and bias estimation. Biometrika 83, 727-745.

Efron, B., Hastie, T., Johnstone, I. \& R. Tibshirani (2004): Least angle regression. Annals of Statistics 32, 407-499.

Fan, J. \& R. Li (2001): Variable selection via nonconcave penalized likelihood and its oracle properties. Journal of the American Statistical Association 96, 1348-1360.

Frank, I. E. \& J. H. Friedman (1993): A statistical view of some chemometrics regression tools (with discussion). Technometrics 35, 109-148.

Kabaila, P. (1995): The effect of model selection on confidence regions and prediction regions. Econometric Theory 11, 537-549.

Knight, K. \& W. Fu (2000): Asymptotics for lasso-type estimators. Annals of Statistics 28, 1356-1378.

Knight, K. (2008): Shrinkage estimation for nearly-singular designs. Econometric Theory 24, forthcoming.

Kulperger, R. J. \& S. E. Ahmed (1992): A bootstrap theorem for a preliminary test estimator. Communications in Statistics: Theory and Methods 21, 2071-2082.

Judge, G. G. \& M. E. Bock (1978): The Statistical Implications of Pre-test and Stein-Rule Estimators in Econometrics. North-Holland.

Leeb, H. \& B. M. Pötscher (2003): The finite-sample distribution of postmodel-selection estimators and uniform versus nonuniform approximations. Econometric Theory 19, 100-142.

Leeb, H. \& B. M. Pötscher (2005): Model selection and inference: Facts and fiction. Econometric Theory 21, 21-59.

Leeb, H. \& B. M. Pötscher (2006a): Performance limits for estimators of the risk or distribution of shrinkage-type estimators, and some general lower risk-bound results. Econometric Theory 22, 69-97. (Corrigendum: ibidem, 24, 581-583).

Leeb, H. \& B. M. Pötscher (2006b): Can one estimate the conditional distribution of post-model-selection estimators? Annals of Statistics 34, 2554-2591.

Leeb, H. \& B. M. Pötscher (2008a): Sparse estimators and the oracle property, or the return of Hodges' estimator. Journal of Econometrics 142, 201-211.

Leeb, H. \& B. M. Pötscher (2008b): Can one estimate the unconditional distribution of post-model-selection estimators? Econometric Theory 24, 338376.

Lehmann, E. L. \& G. Casella (1998): Theory of Point Estimation. Springer Texts in Statistics. New York: Springer-Verlag. 
Pötscher, B. M. (1991): Effects of model selection on inference. Econometric Theory 7, 163-185.

Pötscher, B. M. (2006): The distribution of model averaging estimators and an impossibility result regarding its estimation. IMS Lecture Notes-Monograph Series 52, 113-129.

Pötscher, B. M. \& U. Schneider (2009): On the distribution of the adaptive lasso estimator. Journal of Statistical Planning and Inference, forthcoming, doi:10.1016/j.jspi.2009.01.003.

Samworth, R. (2003): A note on methods of restoring consistency of the bootstrap. Biometrika 90, 985-990.

Sen, P. K. (1979): Asymptotic properties of maximum likelihood estimators based on conditional specification. Annals of Statistics 7, 1019-1033.

Tibshirani, R. (1996): Regression shrinkage and selection via the lasso. Journal of the Royal Statistical Society Series B 58, 267-288.

Zhao, P. \& B. Yu (2006): On model selection consistency of lasso. Journal of Machine Learning Research 7, 2541-2563.

Zou, H. (2006): The adaptive lasso and its oracle properties. Journal of the American Statistical Association 101, 1418-1429. 